\title{
Exploring Cosmic Origins with CORE: Extragalactic Sources in Cosmic Microwave Background Maps
}

G. De Zotti, ${ }^{1}$ J. González-Nuevo, ${ }^{2}$ M. Lopez-Caniego, ${ }^{3} \mathrm{M}$. Negrello, ${ }^{4}$ J. Greenslade, ${ }^{5}$ C. Hernández-Monteagudo, ${ }^{6} \mathrm{~J}$. Delabrouille, ${ }^{7}$ Z.-Y. Cai, ${ }^{8}$ M. Bonato, ${ }^{25,79}$ A. Achúcarro, ${ }^{9,10}$ P. Ade, ${ }^{4}$ R. Allison, ${ }^{74}$ M. Ashdown, ${ }^{48,49}$ M. Ballardini, ${ }^{11,12,13}$ A. J. Banday, ${ }^{14}$ R. Banerji, ${ }^{7}$ J.G. Bartlett, ${ }^{7}$ N. Bartolo, ${ }^{15,16,1}$ S. Basak, ${ }^{18,79}$ M. Bersanelli, ${ }^{19,20}$ M. Biesiada, ${ }^{21}$ M. Bilicki, ${ }^{22,23,45}$ A. Bonaldi, ${ }^{24}$ L. Bonavera, ${ }^{2}$ J. Borrill, ${ }^{26,27} \mathrm{~F}$. Bouchet, ${ }^{28} \mathrm{~F}$. Boulanger, ${ }^{58} \mathrm{~T}$. Brinckmann, ${ }^{29} \mathrm{M}$. Bucher, ${ }^{7} \mathrm{C}$. Burigana, ${ }^{12,30,13}$ A. Buzzelli, ${ }^{35,85}$ M. Calvo, ${ }^{68}$ C. S. Carvalho, ${ }^{32}$ M. G. Castellano, ${ }^{77}$ A. Challinor, ${ }^{17,49,74}$ J. Chluba, ${ }^{24}$ D.L. Clements, ${ }^{5}$ S. Clesse, ${ }^{33}$ S. Colafrancesco, ${ }^{34}$ I. Colantoni, ${ }^{77}$ A. Coppolecchia, ${ }^{35,36}$ M. Crook, ${ }^{78}$ G. D'Alessandro, ${ }^{35}$ P. de Bernardis, ${ }^{35,36}$ G. de Gasperis, ${ }^{31,85}$ J.M. Diego, ${ }^{37}$ E. Di Valentino, ${ }^{28,38}$ J. Errard, ${ }^{39}$ S. M. Feeney, ${ }^{5,81}$ R. Fernández-Cobos, ${ }^{37} \mathrm{~S}$. Ferraro, ${ }^{40} \mathbf{F}$. Finelli, ${ }^{12,13}$ F. Forastieri, ${ }^{41}$ S. Galli, ${ }^{28}$ R.T. Génova-Santos, ${ }^{42,43}$ M. Gerbino, ${ }^{44}$ S. Grandis, ${ }^{46,47}$ S. Hagstotz, ${ }^{46,47}$ S. Hanany, ${ }^{75}$ W. Handley, ${ }^{48,49}$ C. Hervias-Caimapo, ${ }^{24}$ M. Hills, ${ }^{78}$ E. Hivon, ${ }^{28} \mathrm{~K}$. Kiiveri, ${ }^{51,52}$ T. Kisner, ${ }^{26}$ T. Kitching, ${ }^{82}$ M. Kunz, ${ }^{53} \mathrm{H}$. Kurki-Suonio, ${ }^{51,52}$ G. Lagache, ${ }^{54}$ L. Lamagna, ${ }^{35}$ A. Lasenby, ${ }^{48,49}$ M. Lattanzi, ${ }^{41}$ A. Le Brun, ${ }^{55}$ J. Lesgourgues, ${ }^{56}$ A. Lewis, ${ }^{83} \mathrm{M}$. Liguori, ${ }^{15,16,1}$ V. Lindholm, ${ }^{51,52}$ G. Luzzi, ${ }^{31}$ B. Maffei, ${ }^{58}$ N. Mandolesi, ${ }^{30,12}$ E. Martinez-Gonzalez, ${ }^{37}$ C.J.A.P. Martins, ${ }^{57}$ S. Masi, ${ }^{35,36}$ M. Massardi, ${ }^{59}$ D. McCarthy, ${ }^{76}$ A. Melchiorri, ${ }^{35,36}$ J.-B. Melin, ${ }^{60}$ D. Molinari, ${ }^{30,41,12}$ A. Monfardini, ${ }^{68}$ P. Natoli, ${ }^{30,41}$ A. Notari, ${ }^{61}$ A. Paiella, ${ }^{35,36}$ D. Paoletti, ${ }^{12,13}$ R.B. Partridge, ${ }^{62}$ G. Patanchon, ${ }^{7}$ M. Piat, ${ }^{7}$ G. Pisano, ${ }^{4}$ L. Polastri, ${ }^{30,41}$ G. 
Polenta, ${ }^{63,64}$ A. Pollo, ${ }^{65}$ V. Poulin, ${ }^{66,56}$ M. Quartin, ${ }^{67,84}$ M. Remazeilles, ${ }^{24}$ M. Roman, ${ }^{80}$ G. Rossi, ${ }^{86}$ B. F. Roukema, ${ }^{69}$ J.-A. Rubiño-Martín, ${ }^{42,43}$ L. Salvati, ${ }^{35,36}$ D. Scott, ${ }^{70}$ S. Serjeant, ${ }^{71}$ A. Tartari, ${ }^{7}$ L. Toffolatti, ${ }^{2,12}$ M. Tomasi, ${ }^{19,20}$ N. Trappe, ${ }^{76}$ S. Triqueneaux, ${ }^{68}$ T. Trombetti, ${ }^{12,30,13} \mathrm{M}$. Tucci, ${ }^{53} \mathrm{C}$. Tucker, ${ }^{4} \mathrm{~J}$. Väliviita, ${ }^{51,52}$ R. van de Weygaert, ${ }^{50}$ B. Van Tent, ${ }^{72}$ V. Vennin, ${ }^{73}$ P. Vielva, ${ }^{37}$ N. Vittorio, ${ }^{31,85}$ K. Young, ${ }^{75}$ M. Zannoni, ${ }^{87,88}$ for the CORE collaboration

\footnotetext{
${ }^{1}$ INAF-Osservatorio Astronomico di Padova, Vicolo dell'Osservatorio 5, I-35122 Padova, Italy

${ }^{2}$ Departamento de Física, Universidad de Oviedo, C. Calvo Sotelo s/n, 33007 Oviedo, Spain

${ }^{3}$ European Space Agency, ESAC, Planck Science Office, Camino bajo del Castillo, s/n, Urbanización Villafranca del Castillo, Villanueva de la Cañada, Madrid, Spain

${ }^{4}$ School of Physics and Astronomy, Cardiff University, The Parade, Cardiff CF24 3AA, UK

${ }^{5}$ Astrophysics Group, Imperial College, Blackett Laboratory, Prince Consort Road, London SW7 2AZ, UK

${ }^{6}$ Centro de Estudios de Física del Cosmos de Aragón (CEFCA), Plaza San Juan, 1, planta 2, E-44001, Teruel, Spain

${ }^{7}$ APC, AstroParticule et Cosmologie, Université Paris Diderot, CNRS/IN2P3, CEA/lrfu, Observatoire de Paris, Sorbonne Paris Cité, 10, rue Alice Domon et Léonie Duquet, 75205 Paris Cedex 13, France

${ }^{8}$ CAS Key Laboratory for Research in Galaxies and Cosmology, Department of Astronomy, University of Science and Technology of China, Hefei, Anhui 230026, China

${ }^{9}$ Instituut-Lorentz for Theoretical Physics, Universiteit Leiden, 2333 CA, Leiden, The Netherlands

${ }^{10}$ Department of Theoretical Physics, University of the Basque Country UPV/EHU, 48040 Bilbao, Spain

${ }^{11}$ DIFA, Dipartimento di Fisica e Astronomia, Università di Bologna, Viale Berti Pichat, 6/2, I-40127 Bologna, Italy

12 INAF/IASF Bologna, via Gobetti 101, I-40129 Bologna, Italy

13 INFN, Sezione di Bologna, Via Irnerio 46, I-40127 Bologna, Italy

${ }^{14}$ Université de Toulouse, UPS-OMP, IRAP, F-31028 Toulouse cedex 4, France, and CNRS, IRAP, 9 Av. colonel Roche, BP 44346, F-31028 Toulouse cedex 4, France

${ }^{15}$ DIFA, Dipartimento di Fisica e Astronomia “Galileo Galilei”, Università degli Studi di Padova, Via Marzolo 8, I-35131, Padova, Italy

${ }^{16}$ INFN, Sezione di Padova, Via Marzolo 8, I-35131 Padova, Italy

${ }^{17}$ DAMTP, Centre for Mathematical Sciences, University of Cambridge, Wilberforce Road, Cambridge, CB3 0WA, UK

${ }^{18}$ Department of Physics, Amrita School of Arts \& Sciences, Amritapuri, Amrita Vishwa Vidyapeetham, Amrita University, Kerala 690525 India

19 Dipartimento di Fisica, Università degli Studi di Milano, Via Celoria 16, I-20133 Milano, Italy

${ }^{20}$ INAF-IASF, Via Bassini 15, I-20133 Milano, Italy

${ }^{21}$ Department of Astrophysics and Cosmology, Institute of Physics, University of Silesia, Uniwersytecka 4, 40-007 Katowice, Poland

${ }^{22}$ Leiden Observatory, Leiden University, P.O. Box 9513 NL-2300 RA Leiden, The Netherlands

${ }^{23}$ Janusz Gil Institute of Astronomy, University of Zielona Góra, ul. Lubuska 2, 65-265 Zielona Góra, Poland

${ }^{24}$ Jodrell Bank Centre for Astrophysics, School of Physics and Astronomy,

The University of Manchester, Oxford Road, Manchester M13 9PL, UK

${ }^{25}$ Department of Physics \& Astronomy, Tufts University, 574 Boston Avenue, Medford, MA, USA

${ }^{26}$ Computational Cosmology Center, Lawrence Berkeley National Laboratory, Berkeley, California, U.S.A.
} 
27 Space Sciences Laboratory, University of California, Berkeley, California, U.S.A.

${ }^{28}$ Institut d'Astrophysique de Paris (UMR7095: CNRS \& UPMC-Sorbonne Universities), F-75014, Paris, France

${ }^{29}$ Institute for Theoretical Particle Physics and Cosmology (TTK), RWTH Aachen University, D-52056 Aachen, Germany.

${ }^{30}$ Dipartimento di Fisica e Scienze della Terra, Università di Ferrara, Via Giuseppe Saragat 1, I-44122 Ferrara, Italy

31 Dipartimento di Fisica, Università di Roma "Tor Vergata", Via della Ricerca Scientifica 1, I-00133, Roma, Italy

${ }^{32}$ Institute of Astrophysics and Space Sciences, University of Lisbon, Tapada da Ajuda, 1349-018 Lisbon, Portugal

${ }^{33}$ Institute for Theoretical Particle Physics and Cosmology (TTK), RWTH Aachen University, D-52056 Aachen, Germany

${ }^{34}$ School of Physics, Wits University, Johannesburg, South Africa

35 Dipartimento di Fisica, Università di Roma "La Sapienza", Piazzale Aldo Moro 5, I-00185 Roma, Italy

${ }^{36}$ INFN, Sezione di Roma 1, Roma, Italy

37 Instituto de Física de Cantabria (CSIC-UC), Avda. los Castros s/n, 39005 Santander, Spain

38 Sorbonne Universités, Institut Lagrange de Paris (ILP), F-75014, Paris, France

${ }^{39}$ Institut Lagrange, LPNHE, place Jussieu 4, 75005 Paris, France.

${ }^{40}$ Miller Institute for Basic Research in Science, University of California, Berkeley, CA, 94720, USA

${ }^{41}$ INFN, Sezione di Ferrara, Via Saragat 1, 44122 Ferrara, Italy

${ }^{42}$ Instituto de Astrofísica de Canarias, C/Vía Láctea s/n, La Laguna, Tenerife, Spain

${ }^{43}$ Departamento de Astrofísica, Universidad de La Laguna (ULL), La Laguna, Tenerife, 38206 Spain

${ }^{44}$ The Oskar Klein Centre for Cosmoparticle Physics, Department of Physics, Stockholm University, AlbaNova, SE-106 91 Stockholm, Sweden

${ }^{45}$ National Centre for Nuclear Research, Astrophysics Division, P.O. Box 447, PL-90-950 Lodz, Poland

${ }^{46}$ Faculty of Physics, Ludwig-Maximilians Universität, Scheinerstrasse 1, D-81679 Munich, Germany

${ }^{47}$ Excellence Cluster Universe, Boltzmannstr. 2, D-85748 Garching, Germany

${ }^{48}$ Astrophysics Group, Cavendish Laboratory, Cambridge, CB3 0HE, UK

${ }^{49}$ Kavli Institute for Cosmology, Madingley Road, Cambridge, CB3 0HA, UK

${ }^{50}$ Kapteyn Astronomical Institute, University of Groningen, P.O. Box 800, 9700AV, Groningen, the Netherlands

${ }^{51}$ Department of Physics, Gustaf Hällströmin katu 2a, University of Helsinki, Helsinki, Finland

${ }^{52}$ Helsinki Institute of Physics, Gustaf Hällströmin katu 2, University of Helsinki, Helsinki, Finland

${ }^{53}$ Département de Physique Théorique and Center for Astroparticle Physics, Université de Genève, 24 quai Ansermet, CH-1211 Genève 4, Switzerland

${ }^{54}$ Aix Marseille Université, CNRS, LAM (Laboratoire d'Astrophysique de Marseille) UMR 7326, 13388, Marseille, France

${ }^{55}$ Laboratoire AIM, IRFU/Service d'Astrophysique - CEA/DRF - CNRS - Université Paris Diderot, Bât. 709, CEASaclay, 91191 Gif-sur-Yvette Cedex, France

${ }^{56}$ Institute for Theoretical Particle Physics and Cosmology (TTK), RWTH Aachen University, D-52056 Aachen, Germany.

${ }^{57}$ Centro de Astrofísica da Universidade do Porto and IA-Porto, Rua das Estrelas, 4150-762 Porto, Portugal

${ }^{58}$ Institut d'Astrophysique Spatiale, CNRS, UMR 8617, Université Paris-Sud 11, Bâtiment 121, 91405 Orsay, France

${ }^{59}$ INAF, Osservatorio di Radioastronomia, Via Gobetti 101, I-40129, Bologna

${ }^{60}$ CEA Saclay, DRF/Irfu/SPP, 91191 Gif-sur-Yvette Cedex, France

${ }^{61}$ Departamento de Física Quàntica i Astrofísica i Institut de Ciències del Cosmos, Universitat de Barcelona, Martíi Franquès 1, 08028 Barcelona, Spain 
${ }^{62}$ Department of Physics and Astronomy, Haverford College, Haverford, PA, USA 19041

${ }^{63}$ Agenzia Spaziale Italiana Science Data Center, Via del Politecnico snc, 00133, Roma, Italy

${ }^{64}$ INAF - Osservatorio Astronomico di Roma, via di Frascati 33, Monte Porzio Catone, Italy

${ }^{65}$ National Center for Nuclear Research, ul. Hoża 69, 00-681 Warsaw, Poland, and The Astronomical Observatory of the Jagiellonian University, ul. Orla 171, 30-244 Kraków, Poland

${ }^{66}$ LAPTh, Université Savoie Mont Blanc \& CNRS, BP 110, F-74941 Annecy-le-Vieux Cedex, France

${ }^{67}$ Instituto de Física, Universidade Federal do Rio de Janeiro, 21941-972, Rio de Janeiro, Brazil

${ }^{68}$ Institut Néel, CNRS and Université Grenoble Alpes, F-38042 Grenoble, France

${ }^{69}$ Toruń Centre for Astronomy, Faculty of Physics, Astronomy and Informatics, Grudziadzka 5, Nicolaus Copernicus University, ul. Gagarina 11, 87-100 Toruń, Poland

${ }^{70}$ Department of Physics and Astronomy, University of British Columbia, Vancouver, BC, Canada V6T1Z1

${ }^{71}$ School of Physical Sciences, The Open University, Walton Hall, Milton Keynes MK7 6AA, UK

72 Laboratoire de Physique Théorique (UMR 8627), CNRS, Université Paris-Sud, Université Paris Saclay, Bâtiment 210 , 91405 Orsay Cedex, France

${ }^{73}$ Institute of Cosmology and Gravitation, University of Portsmouth, Dennis Sciama Building, Burnaby Road, Portsmouth PO1 3FX, United Kingdom

${ }^{74}$ Institute of Astronomy, Madingley Road, Cambridge, CB3 0HA, UK

${ }^{75}$ School of Physics and Astronomy and Minnesota Institute for Astrophysics, University of Minnesota/Twin Cities, USA

${ }^{76}$ Department of Experimental Physics, Maynooth University, Maynooth, Co. Kildare, W23 F2H6, Ireland

${ }^{77}$ Istituto di Fotonica e Nanotecnologie - CNR, Via Cineto Romano 42, I-00156 Roma, Italy

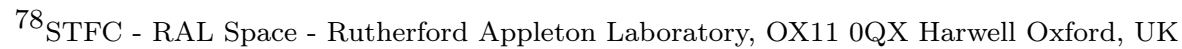

${ }^{79}$ SISSA, Via Bonomea 265, 34136, Trieste, Italy

${ }^{80}$ Laboratoire de Physique Nucléaire et des Hautes Énergies (LPNHE), Université Pierre et Marie Curie, Paris, France

${ }^{81}$ Center for Computational Astrophysics, 160 5th Avenue, New York, NY 10010, USA

${ }^{82}$ Mullard Space Science Laboratory, University College London, Holmbury St Mary, Dorking, Surrey RH5 6NT, UK

83 Department of Physics \& Astronomy, University of Sussex, Brighton BN1 9QH, UK

${ }^{84}$ Observatório do Valongo, Universidade Federal do Rio de Janeiro, Ladeira Pedro Antônio 43, 20080-090, Rio de Janeiro, Brazil

${ }^{85}$ Sezione INFN Roma 2, Via della Ricerca Scientifica 1, I-00133, Roma, Italy

${ }^{86}$ Department of Astronomy and Space Science, Sejong University, Seoul 143-747, Korea

${ }^{87}$ Dipartimento di Fisica, Università di Milano Bicocca, Milano, Italy

${ }^{88}$ INFN, Sezione di Milano Bicocca, Milano, Italy

E-mail: gianfranco.dezotti@oapd.inaf.it

Abstract. We discuss the potential of a next generation space-borne Cosmic Microwave Background (CMB) experiment for studies of extragalactic sources. Our analysis has particular bearing on the definition of the future space project, CORE, that has been submitted in response to ESA's call for a Medium-size mission opportunity as the successor of the Planck satellite. Even though the effective telescope size will be somewhat smaller than that of Planck, CORE will have a considerably better angular resolution at its highest frequencies, since, in contrast with Planck, it will be diffraction limited at all frequencies. The improved resolution implies a considerable decrease of the source confusion, i.e. substantially fainter detection limits. In particular, CORE will detect thousands of strongly lensed high- $z$ galaxies 
distributed over the full sky. The extreme brightness of these galaxies will make it possible to study them, via follow-up observations, in extraordinary detail. Also, the CORE resolution matches the typical sizes of high- $z$ galaxy proto-clusters much better than the Planck resolution, resulting in a much higher detection efficiency; these objects will be caught in an evolutionary phase beyond the reach of surveys in other wavebands. Furthermore, CORE will provide unique information on the evolution of the star formation in virialized groups and clusters of galaxies up to the highest possible redshifts. Finally, thanks to its very high sensitivity, CORE will detect the polarized emission of thousands of radio sources and, for the first time, of dusty galaxies, at $\mathrm{mm}$ and sub-mm wavelengths, respectively.

Keywords: cosmology: observations - surveys - submillimeter: galaxies - radio continuum: general - galaxies: evolution

\section{Introduction}

Although not specifically designed for the observation of extragalactic sources, space-borne experiments aimed at investigating the Cosmic Microwave Background (CMB) have the potential to bring breakthroughs also in this field. An investigation of the impact on studies of extragalactic sources of the project named the Cosmic Origins Explorer plus (COrE + ), submitted in response to ESA's call for the 4th Medium-size mission (M4) opportunity, was carried out by Ref. [1]. Various options were considered, with effective telescope sizes of $\simeq 1 \mathrm{~m}, 1.5 \mathrm{~m}$ and $2 \mathrm{~m}$, and a frequency range from 60 to $1200 \mathrm{GHz}$. A proposal for ESA's 5th Medium-size mission (M5) is envisaging an instrument (named CORE) with a baseline telescope size of $1.2 \mathrm{~m}$ and 19 frequency channels, distributed over the $60-600 \mathrm{GHz}$ frequency range. A decrease or an increase of the telescope size to $1 \mathrm{~m}$ and to $1.5 \mathrm{~m}$, respectively, were also considered. These options will be referred to as CORE100 and CORE150. For the CORE 150 configuration we will also consider the added value of an extension of the frequency range to $800 \mathrm{GHz}$.

Since the analysis by Ref.[1] was completed, considerable relevant new data have become available and more detailed studies have been carried out, motivating an update for the 5th Medium-size mission (M5) proposal. In particular, most analyses of the Planck data have now been published, giving much clearer predictions for the capabilities of next generation CMB experiments.

The plan of the paper is the following. In Sect. 2 we present a new assessment of the expected counts of the various classes of extragalactic sources in total intensity. In Sect. 3 we highlight the CORE potential for detecting galaxy proto-clusters during their early evolutionary phase when they did not yet possess the hot intergalactic medium allowing detection via their X-ray emission and/or the Sunyaev-Zeldovich (SZ) effect. As shown in Sect. 4, CORE will also provide unique information on the evolution of the star-formation rate (SFR) in virialized clusters. Section 5 deals with the information provided by CORE surveys on the Cosmic Infrared Background (CIB), while the effect of bright sub-mm lines on the power spectra measured in different frequency intervals and the possibility of counts being estimated in lines are considered in Sect. 6. In Sect. 7 we discuss counts in polarized flux density and report the results of new simulations aimed at determining the CORE detection limits in polarization, showing that CORE will provide a real breakthrough in this field. Our main conclusions are summarized in Sect. 8. 
Table 1. Estimated $4 \sigma$ CORE detection limits, $S_{\mathrm{d}}(\mathrm{mJy})$, for 4 effective telescope sizes. The values of $S_{\mathrm{d}}$ were derived from the simulations described in Ref. [1] and refer to regions of low Galactic emission.

\begin{tabular}{rrrrr}
\hline \hline$\nu(\mathrm{GHz})$ & $1 \mathrm{~m}$ & $1.2 \mathrm{~m}$ & $1.5 \mathrm{~m}$ & $2 \mathrm{~m}$ \\
\hline 60 & 197.9 & 147.1 & 94.4 & 55.3 \\
70 & 200.1 & 149.5 & 94.8 & 55.3 \\
80 & 197.1 & 148.1 & 92.7 & 53.9 \\
90 & 190.5 & 144.2 & 89.1 & 51.6 \\
100 & 182.0 & 138.7 & 84.8 & 49.0 \\
115 & 169.5 & 130.7 & 78.6 & 45.2 \\
130 & 156.7 & 122.2 & 72.5 & 41.7 \\
145 & 144.7 & 114.0 & 66.9 & 38.4 \\
160 & 131.8 & 105.3 & 61.0 & 35.1 \\
175 & 119.2 & 96.6 & 55.2 & 31.9 \\
195 & 104.9 & 86.9 & 49.0 & 28.8 \\
220 & 91.6 & 78.1 & 43.8 & 26.4 \\
255 & 80.7 & 70.9 & 41.1 & 26.0 \\
295 & 81.0 & 73.1 & 44.1 & 29.1 \\
340 & 90.5 & 83.2 & 51.5 & 34.9 \\
390 & 104.5 & 97.1 & 60.7 & 41.6 \\
450 & 121.8 & 113.7 & 71.3 & 49.3 \\
520 & 140.7 & 131.5 & 82.5 & 57.6 \\
600 & 150.5 & 139.8 & 90.4 & 63.5 \\
\hline \hline
\end{tabular}

Throughout this paper we adopt the fiducial $\Lambda$ CDM cosmology with the best-fit values of the parameters derived from Planck CMB power spectra, in combination with lensing reconstruction and external data: $H_{0}=67.74 \mathrm{~km} \mathrm{~s}^{-1} \mathrm{Mpc}^{-1} ; \Omega_{\Lambda}=0.6911 ;$ and $\Omega_{\mathrm{m}}=0.3081$ [2].

This work is part of a series of papers that present the science achievable by the CORE space mission. The performance requirements and the mission concept are described in [3]. The instrument is described in [4]. Reference [5] explores systematic effects that may represent a threat to the measurement accuracy. Reference [6] discusses polarised foregrounds and the $B$-mode component separation. The constraints on cosmological parameters and fundamental physics that can be derived from CORE measurements are discussed in [7] while the constraints on inflationary models are discussed in [8]. References [9] and [10] deal large-scale structure and cluster science, respectively, while [11] addresses the effect on the CMB of the observer's peculiar motion.

\section{Counts of extragalactic sources in total intensity}

As pointed out by Ref. [1], CORE surveys will be confusion limited. This was already the case for the Planck High Frequency Instrument (HFI) which however reached the diffraction limit only up to $217 \mathrm{GHz}$, while CORE will be diffraction limited over its full frequency range. Since the confusion limit scales roughly as the beam solid angle, i.e. as the square of the full width at half maximum (FWHM) of the instrument [see figure 3 of Ref. 1], CORE will 
substantially improve over Planck-HFI, even in the case of a somewhat smaller telescope. For example, at $545 \mathrm{GHz}(550 \mu \mathrm{m})$ the Planck beam has an effective $\mathrm{FWHM}=4.83^{\prime}$, while the diffraction limit for its $1.5-\mathrm{m}$ telescope is $1.5^{\prime}$. Realistic simulations [1] gave, at this frequency, $4 \sigma$ completeness limits of $86 \mathrm{mJy}$ for the CORE $1.5 \mathrm{-m}$ option and of $142 \mathrm{mJy}$ for the 1-m option. For comparison the $90 \%$ completeness limit for the Second Planck Catalog of Compact Sources [PCCS 2; 12] at $545 \mathrm{GHz}$ is $555 \mathrm{mJy}$.

At the lower frequencies covered by the Planck Low Frequency Instrument (LFI), whose resolution was also at the diffraction limit, CORE performs better thanks to its lower instrumental noise. For example at $70 \mathrm{GHz}$ the PCCS $290 \%$ completeness limit is $501 \mathrm{mJy}$, while simulations give, for CORE, $4 \sigma$ completeness limits of $95 \mathrm{mJy}$ and $200 \mathrm{mJy}$ for the $1.5-\mathrm{m}$ and 1-m options, respectively [1].

Reference [1] found that the $4 \sigma \mathrm{CORE}$ detection limits, $S_{\mathrm{d}}$, derived from realistic simulations, are well approximated by the formula:

$$
S_{\mathrm{d}}=4\left[\sigma_{\text {conf }}^{2}+\sigma_{\text {noise }}^{2}+\left(0.12 \sigma_{\mathrm{CMB}}\right)^{2}\right]^{1 / 2},
$$

with

$$
\sigma_{\text {conf }}^{2}=\sigma_{\mathrm{P}, \text { radio }}^{2}+\sigma_{\mathrm{P}, \text { dusty }}^{2}+\sigma_{\text {clust,dusty }}^{2}+\sigma_{\mathrm{SZ}}^{2} .
$$

Here, $\sigma_{\text {conf }}$ and $\sigma_{\mathrm{CMB}}$ are the rms fluctuations at the instrument resolution due source confusion and to CMB anisotropies, respectively, computed using the formulae in Sect. 2.1 of Ref. [1]. The source confusion term, due to sources below the detection limit, includes contributions of Poisson fluctuations due to radio sources, $\sigma_{\mathrm{P} \text {,radio }}$, and to dusty galaxies $\sigma_{\mathrm{P}, \text { dusty }}$, as well as the contribution of clustering of dusty galaxies, $\sigma_{\text {clust,dusty }}$ (the contribution of clustering of radio sources was found to be negligibly small). The contribution of CORE instrumental noise, $\sigma_{\text {noise }}$, turns out to be always almost negligible. A tabulation of $4 \sigma$ CORE detection limits, $S_{\mathrm{d}}$, for the 19 frequency channels and four effective telescope sizes $(1 \mathrm{~m}$, $1.2 \mathrm{~m}, 1.5 \mathrm{~m}$, and $2 \mathrm{~m}$ ) is given in Table 1 .

\section{$2.1 \quad$ Local dusty galaxies}

The Planck surveys offered the first opportunity to accurately determine the luminosity function of dusty galaxies in the very local universe at several (sub-)millimetre wavelengths, using blindly selected samples of low-redshift sources, unaffected by cosmological evolution [13]. At 857,545 and $353 \mathrm{GHz}$ the local luminosity function could be determined over about three decades in luminosity. Remarkably, Planck reached luminosities almost one order of magnitude fainter than Herschel's Spectral and Photometric Imaging REceiver (SPIRE) surveys at the common or nearby frequencies $(857$ and $545 \mathrm{GHz}$, corresponding to wavelengths of 350 and $550 \mu \mathrm{m}$ ) [14]: Planck's all-sky coverage has proven to be much more effective in detecting local low-luminosity sub-mm galaxies than Herschel-SPIRE, in spite of the much deeper flux density levels reached by the latter instrument, on small sky areas.

Estimates of the CORE detection limits [1] show that it will reach, at 500-600 GHz, flux densities a factor of 5 (1-m option) or 8.6 (1.5-m option) fainter than the PCCS2. CORE will thus explore a volume larger by a factor of $\simeq 11$ or $\simeq 25$, respectively, thus improving the source statistics by similar factors. As a result, $\mathrm{CORE}$ will detect, at $600 \mathrm{GHz}$ and with $\geq 4 \sigma$ significance, from $\simeq 14,000$ (1-m option) to $\simeq 30,600$ (1.5-m option) star-forming galaxies out to $z=0.1$ in the "extragalactic zone" $\left(|b|>30^{\circ}\right)$ defined by Ref. [12]. ${ }^{1}$

\footnotetext{
${ }^{1}$ Note that table 13 of Ref. [12] lists the total number of Planck detections, not only those above the $90 \%$ completeness limit.
} 
Within the timescales of the CORE launch, there will be several wide-angle redshift and photometric surveys available that should provide distance information for the majority, if not all, of the galaxies detected by the satellite. Of most interest of course are data sets covering the entire sky. In the near future spectroscopic data for all the Two Micron All Sky Survey (2MASS) galaxies ( 1 million over most of sky, median $z \sim 0.1$ ) are expected from the TAIPAN survey [15] and the Low Redshift survey at Calar Alto (LoRCA) project [16]. On a longer timescale, the Spectro-Photometer for the History of the Universe, Epoch of Reionization, and Ices Explorer (SPHEREx) mission [17] aims at providing a low-resolution spectroscopic survey of the entire sky in the near-IR, including millions of galaxies up to $z>1$.

Another avenue towards all-sky 3D catalogues is through combing imaging data-sets and deriving photometric redshifts. This was successfully completed first for 2MASS galaxies [2MPZ; 18$]$ and recently also for a combination of WISE and SuperCOSMOS scans of photographic plates [19]. The WISE/SuperCOSMOS catalogue maps the large scale structure (LSS) at $z<0.4$ for $70 \%$ of sky.

The deeper CORE surveys, compared to Planck, coupled with redshift information, will make it possible to extend the determination of the luminosity function of dusty galaxies down to fainter luminosities and to higher redshifts. The information from blind detections on CORE maps can be expanded by exploiting the prior knowledge of galaxy positions from optical/IR surveys to push the detection limits to fainter levels, a technique successfully tested in Ref. [20]. Stacking analyses on positions of known galaxies will allow us to reach still fainter flux densities.

A variety of photometric data will also be available across the electromagnetic spectrum (radio, IRAS, AKARI, WISE, Euclid, GALEX, ROSAT, eROSITA) in addition to multi-band optical/near-IR imaging. Combining them with CORE data, it will be possible to carry out an extensive characterization of the properties of galaxies in the nearby Universe [e.g. 21]. CORE data will be crucial in particular to achieve progress towards understanding how dust mass and emission vary with galaxy type and stellar mass. The combination of the CORE data with data in different wavebands will allow us to determine, for each galaxy type and as a function of stellar mass, the distribution of dust temperatures, as well as the dust mass function, the SFR function, the relationship between star formation and nuclear activity, the relative contributions of newborn and evolved stars to dust heating, and more.

The next step will be to relate the properties of galaxies detected by CORE to the underlying dark matter field and to the properties of their host dark matter haloes. Local dusty star-forming galaxies are found to be more clustered and to reside in more massive haloes than galaxies with unobscured star formation [22, 23]. CORE will provide an unprecedented sample to investigate the link between local dusty galaxies of different types and their local environments.

At $\mathrm{mm}$ wavelengths we can exploit the synergy with the Stage-IV CMB (CMB-S4) surveys [24]. This next generation ground-based program aims to deploy $\mathrm{O}(500,000)$ detectors spanning the $30-300 \mathrm{GHz}$ frequency range. It will use multiple telescopes and sites to map most of the sky and is expected to be operational between about 2021 and 2024. Thanks to its higher sensitivity, the CMB-S4 experiment will extend to longer wavelengths the spectral coverage of dusty galaxies detected by CORE at sub-mm wavelengths. Among other things, this will allow us to test the hints from Planck that some fraction of local galaxies have a significant very cold dust component [10-13 K; 25] which the mm bands of CMB-S4 will be especially powerful in detecting. 

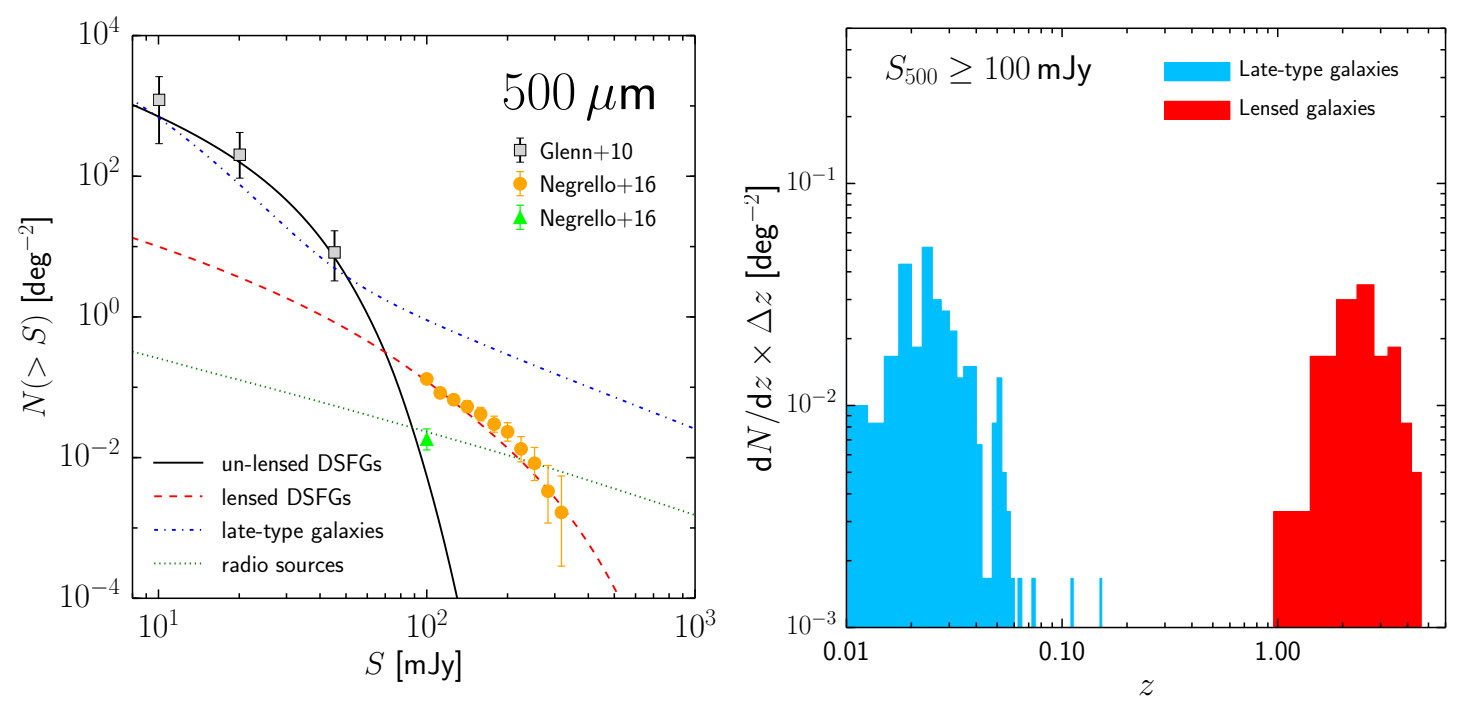

Figure 1. Left panel. Counts of candidate gravitationally-lensed dusty star-forming galaxies (DSFGs) from the Herschel Astrophysical Terahertz Large Area Survey (H-ATLAS), over an area of about $600 \mathrm{deg}^{2}$ [26, filled orange circles], compared with the counts of unlensed high- $z$ DSFGs, interpreted as proto-spheroidal galaxies [solid black curve; 27], of normal late-type and starburst galaxies (referred to as "late-type galaxies" in the inset; dot-dashed blue line) and of radio sources (blazars, dotted green line; the data point, green triangle, is from Ref. [26]). The dashed red line shows the model counts of strongly lensed galaxies for a maximum magnification $\mu_{\max }=10$ [26]; rare much higher magnifications can be produced by cluster lensing. The counts of normal late-type, starburst and proto-spheroidal galaxies have been computed using the model described in Ref. [28]. The effect of lensing has been computed following Ref. [29]. The counts of radio sources are from Ref. [30]. Right panel. Redshift distribution of galaxies brighter than $100 \mathrm{mJy}$ at $500 \mu \mathrm{m}$, derived from the full H-ATLAS catalogue [26]. There is a clear bimodality. On one side we have nearby late-type galaxies, almost all at $z \leq 0.06$, and hence easily recognizable in optical/near-infrared catalogues. On the other side we have dust enshrouded, hence optically very faint, gravitationally lensed galaxies at $z \geq 1$ and up to $z>4$.

\subsection{High- $z$ dusty galaxies}

At sub-mm wavelengths CORE will reach faint enough flux densities to see the transition from the Euclidean portion of the counts contributed by low- $z$ galaxies to the steeply rising portion caused by cosmological evolution. The precise shape of the counts in this transition region provides critical constraints to evolutionary models [31]. There are in fact two main scenarios discussed in the literature for the evolution of galaxies as viewed at far-IR/sub$\mathrm{mm}$ wavelengths. One scenario envisages a direct evolutionary link between local and high- $z$ galaxies, with no new populations. According to the other scenario, a major component of the dusty galaxy population at high- $z$, is made, for example, by massive proto-spheroidal galaxies in the process of forming the bulk of their stars. These galaxies have no low- $z$ counterparts at far-IR/sub-mm wavelengths because they have been essentially passively evolving since $z \simeq 1-1.5$. Clearly the first scenario implies a more gradual steepening of the slope of the counts than the second one.

CORE will provide a complete census of the brightest sub-mm galaxies in the Universe, including those with extreme amplification by gravitational lensing. Planck has already of- 
fered an exciting foretaste of that: "Planck's dusty GEMS" [Gravitationally Enhanced subMillimetre Sources; 32]. As discussed in Ref. [32], follow-up CO spectroscopy and multi-frequency photometry of 11 such sources have shown that they are at $z=2.2-3.6$ and have apparent (uncorrected for gravitational amplification) far-IR luminosities of up to $3 \times 10^{14} \mathrm{~L}_{\odot}$, making them among the brightest sources in the Universe. They are so rare that only very large area surveys can find them.

Ref. [33] have found 77 candidate strongly lensed galaxies brighter than $100 \mathrm{mJy}$ at $500 \mu \mathrm{m}$ from Herschel surveys covering $372 \mathrm{deg}^{2}$, corresponding to a surface density of $\simeq$ $0.2 \mathrm{deg}^{-2}$. A somewhat lower surface density at the same flux density limit has been derived in Ref. [26] from the Herschel Astrophysical Terahertz Large Area Survey (H-ATLAS); they found 79 candidates over an area of $602 \mathrm{deg}^{2}$. Averaging the two estimates we obtain a surface density of $\simeq 0.16 \mathrm{deg}^{-2}$. The CORE detection limits at $\simeq 500 \mu \mathrm{m}$ are $\simeq 150 \mathrm{mJy}$ and $\simeq 90 \mathrm{mJy}$ for the $1-\mathrm{m}$ and the $1.5-\mathrm{m}$ telescope, respectively. The average surface density of strongly lensed galaxies with $S_{500 \mu \mathrm{m}}>150 \mathrm{mJy}$ is $\simeq 0.0465 \mathrm{deg}^{-2}$ implying that the $1-\mathrm{m}$ option will allow the discovery of about one thousand strongly lensed galaxies in the "extragalactic zone". A slight extrapolation to 90 mJy using the model shown in Fig. 1 leads to an estimate of about 3,000 strongly lensed galaxies detectable by CORE150 in the "extragalactic zone".

At these flux densities the selection of strongly lensed galaxies can be easily done with close to $100 \%$ efficiency $[34,35]$. In fact, as illustrated by Fig. 1, in the relevant flux density range, i.e. above $S_{500 \mu \mathrm{m}} \simeq 90 \mathrm{mJy}$, the dominant populations of extragalactic sources are low- $z$ galaxies and high- $z$ strongly lensed galaxies (see the right-hand panel of the figure). The former are easily recognisable in all-sky optical/near-IR surveys, such as the UK Schmidt Telescope and Second Palomar Observatory survey [see the recent SuperCOSMOS all-sky galaxy catalogue; 36], the Two-Micron All-Sky Survey [2MASS; 37] and the Wide-field Infrared Survey Explorer [WISE; 38]. In addition there is a small fraction of radio sources, mostly blazars, also easily identifiable by cross-matching with all-sky radio surveys, such as the NRAO VLA Sky Survey [NVSS; 39] plus the Sydney University Molonglo Sky Survey [SUMSS; 40].

There will be a large overlap between the strongly lensed galaxies detected by the CMBS4 surveys and those detected by CORE. The two sets of observations will allow a better definition of the SEDs, hence a better photometric redshift estimate, and an improved determination of the source positions, essential for follow-up observation. Due to the shape of the dust emission SED, CORE will be more efficient at detecting objects at $z \lesssim 2$, while the longer wavelength observations by the CMB-S4 will be more effective at detecting those at $z \gtrsim 3$. Note however that the sub-mm CORE measurements will be essential to characterize the emission peak, hence to derive basic quantities like the total IR luminosity, hence the SFR.

These large samples of strongly gravitationally lensed galaxies will be trivially easy targets for ALMA, NOEMA, etc., and the foreground lenses will almost certainly be detectable in, e.g., Euclid imaging. Follow-up observations will allow us to determine the total (visible and dark) mass of the lensing galaxies, to investigate their density profiles, and to measure cosmological parameters and especially the Hubble constant using gravitational time delays [e.g. 41, 42, and references therein]. The time delay distance measurement are estimated to reach an uncertainty of $5-7 \%$ and are independent of the local distance ladder; they thus provide a crucial test of any potential systematic uncertainties. Although the Planck determination of $H_{0}$ from $\mathrm{CMB}$ anisotropies has a much higher precision, it should be noted 
that time delay distances are completely independent of the properties of the early Universe and may thus allow one to break some of the main degeneracies in the interpretation of CMB data.

While other facilities (e.g., Euclid, Gaia, SKA) will also be generating large gravitational lens catalogues on a comparable timescale [see, e.g., 43, for a recent review] the critical advantage of CORE and of CMB-S4 will be in extending the sources and lenses to much higher redshifts and in detecting the most extreme amplifications. This has been demonstrated by Planck: the magnification factors, $\mu$, of "Planck's dusty GEMS" are estimated to be up to 50 [32].

The flux boosting is accompanied by a stretching of images. In general, the image size scales approximately as $\mu$ in one direction (tangentially) while being unchanged in the perpendicular direction (radially). The geometric mean is an overall scaling of $\mu^{1 / 2}$ as demanded by the conservation of surface brightness. The extreme apparent luminosities and the stretching of images allow us to study the properties and internal structure of these high- $z$ sources in extraordinary detail, much greater than would be possible otherwise. The evolution of dark matter halo substructure and the stellar initial mass function (IMF) can thus be probed to much higher redshifts, providing key tests for galaxy evolution theory.

Another important advantage compared to optical/near-IR searches is that foreground lenses and background magnified galaxies stand out in different wavebands. The background lensed galaxies CORE will detect are heavily dust obscured, hence are bright at far-IR/(sub)mm wavelengths but are almost invisible in the optical/near-IR. On the contrary, most foreground lenses are passive spheroidal galaxies, hence bright in the optical/near-IR but almost invisible at far-IR/(sub-)mm wavelengths. Therefore the mutual contamination of their images is small or negligible. This is obviously a major advantage for detailed modelling.

For the unlensed population, the CORE survey will also probe essentially the entire Hubble volume for the most intense hyperluminous starbursts, testing whether there are physical limits to the star-formation rates of galaxies [e.g. Eddington-limited star formation; 44].

\section{Protoclusters of dusty galaxies}

As shown in Ref. [1], at sub-mm wavelengths fluctuations in Planck maps are dominated by the clustering of dusty galaxies at high $z$. This implies that low-resolution surveys in this spectral range are optimally suited for detecting overdensities of star-forming galaxies that may evolve into present day rich clusters [48].

A blind search on Planck maps for candidate high- $z$ proto-clusters was carried out in Ref. [49], looking for intensity peaks with "cold" sub-mm colours, i.e. with continuum spectra peaking between 353 and $857 \mathrm{GHz}$ at $5^{\prime}$ resolution, consistent with redshifts $z>2$ for typical dust emission spectra. Their selection required detections at $\mathrm{S} / \mathrm{N}>3$ in all three highest frequency Planck bands and applied a flux density threshold of $S_{545 \mathrm{GHz}}>500 \mathrm{mJy}$. A total of 2151 high $z$ source candidates satisfying these criteria were selected in the cleanest $26 \%$ of the sky, corresponding to a surface density of $\simeq 0.2 \mathrm{deg}^{-2}$. Apart from a tiny fraction (around $3 \%$ ) of extreme strongly lensed high- $z$ galaxies, these intensity peaks appear to be associated with excess surface densities of high- $z$ dusty star-forming galaxies. This conclusion was substantiated by Herschel/SPIRE follow-up of 234 Planck "cold" targets [50], finding that about $94 \%$ of them indeed show a significant excess of red 350 and $500 \mu \mathrm{m}$ sources in comparison to reference SPIRE fields. 


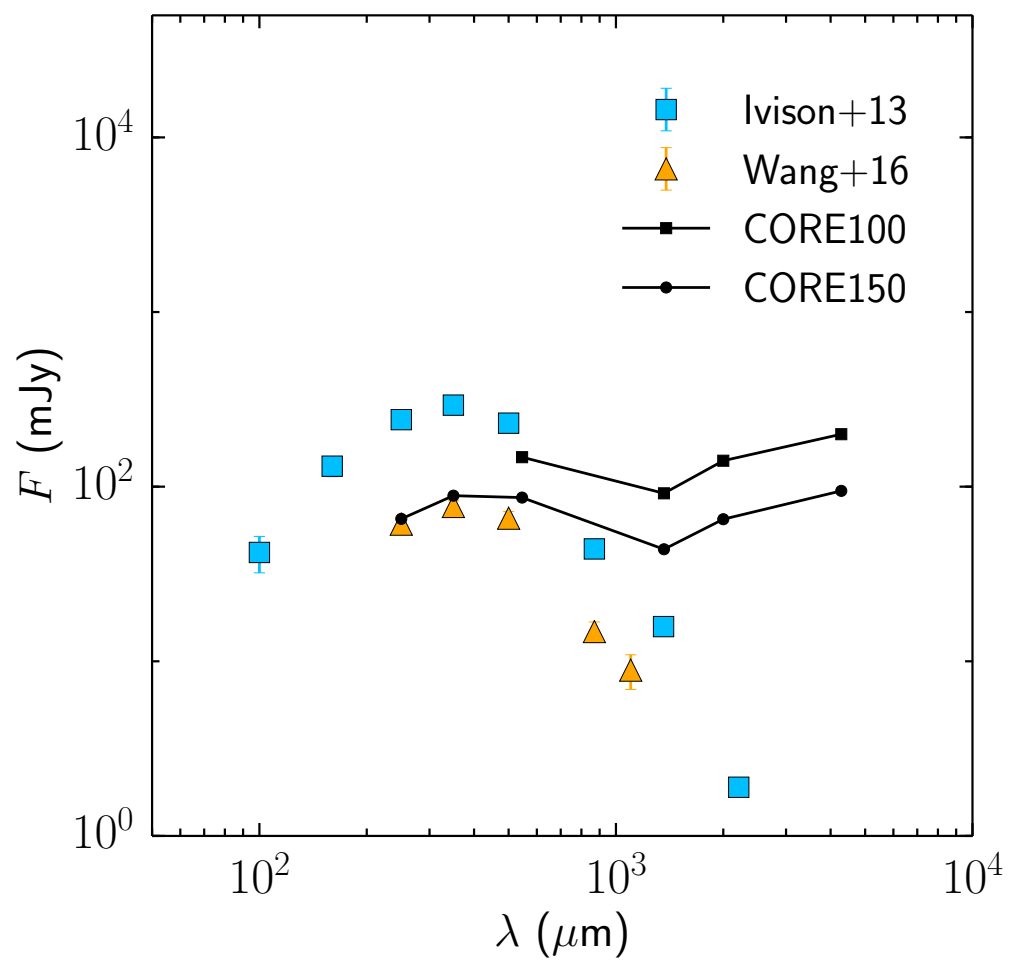

Figure 2. Spectral energy distributions (SEDs) of the cores of two clusters of starbursting galaxies. The blue squares show the SEDs of the proto-cluster core at $z=2.41$ [45] discovered by means of observations with the Jansky Very Large Array (JVLA) of a bright H-ATLAS source that did not show indications of strong lensing. The proto-cluster core has a linear size of approximately $100 \mathrm{kpc}$, i.e. an angular size of about 12 arcsec. This object shows extended X-ray emission, suggesting that it is already virialized; however, it is dominated by star-forming galaxies. The orange triangles show the SED of the proto-cluster core at $z=2.506$ [46] discovered in the COSMOS field. Its size is about $80 \mathrm{kpc}$. The solid black lines show the CORE detection limits for the 1-m and 1.5-m telescope (from top to bottom).

The detected sources have a flux density distribution peaking at $S_{545 \mathrm{GHz}} \simeq 1 \mathrm{Jy}$ and extending up to $S_{545 \mathrm{GHz}} \simeq 2 \mathrm{Jy}$. If they are indeed proto-clusters at $z>2$, they have extraordinarily high total $(8-1000 \mu \mathrm{m})$ infrared luminosities, $L_{\mathrm{IR}}$, peaking around $2 \times 10^{14} L_{\odot}$ for a reference dust temperature of $35 \mathrm{~K}$ [49]. The corresponding star formation rate (SFR) is $\simeq 3 \times 10^{4} \mathrm{yr}^{-1}$ [51]. The associated halo mass, $M_{\mathrm{h}}$, obtained from the $\mathrm{SFR}-M_{\mathrm{h}}$ relation at $z=2$ derived exploiting the abundance matching technique, is $M_{\mathrm{h}} \simeq 5 \times 10^{14} \mathrm{M}_{\odot}$ [52]. But the surface density of halos that massive at $z \simeq 2$ is only $\simeq 5 \times 10^{-5} \mathrm{deg}^{-2}$, well below that of Planck over-densities.

On the other hand, the halo surface density increases steeply with decreasing $M_{\mathrm{h}}$ : already at $M_{\mathrm{h}} \simeq 10^{14} \mathrm{M}_{\odot}$ it reaches $\simeq 0.3 \mathrm{deg}^{-2}$. This suggests that the candidate proto-clusters detected in Ref. [49] may not be individual objects but fluctuations in the number of somewhat smaller, physically unrelated clumps of dusty galaxies along the line of sight. Extensive simulations by Negrello et al. (in preparation) showed that indeed the results of Ref. [49] 

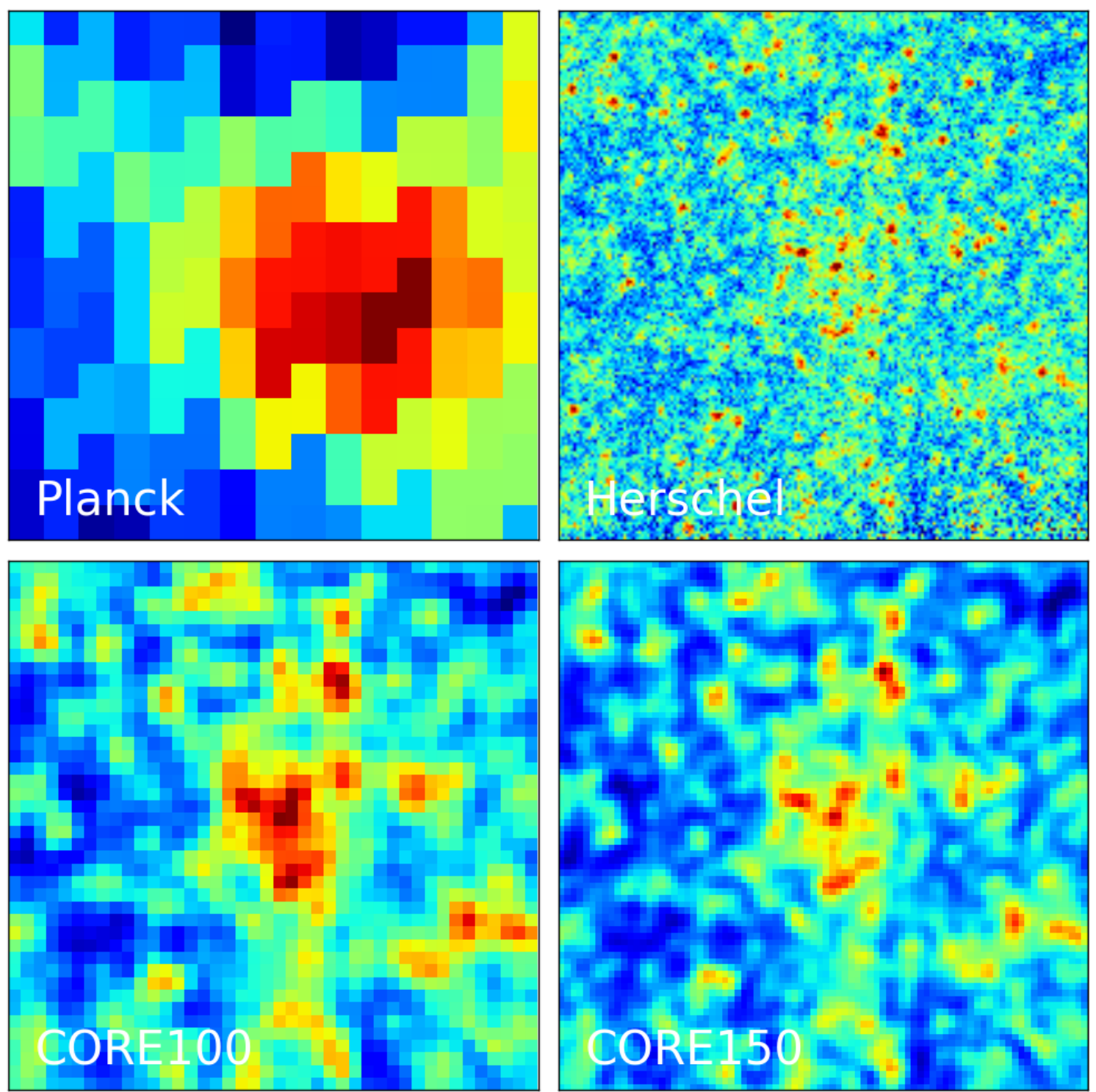

Figure 3. Planck (upper left panel) and Herschel (upper right panel) images at 857 and $600 \mathrm{GHz}$, respectively, of a candidate $z=2.3$ proto-cluster in the Bootes field of the HerMES survey [47], compared with the appearance it may take for the diffraction limited beams of CORE100 and CORE 150 at $600 \mathrm{GHz}$. Pixel sizes for Planck and both CORE options are FWHM/2.517. The size of each map is $25^{\prime} \times 25^{\prime}$.

can be understood in these terms. Support for this view comes from the fact that the only Planck overdensity for which spectroscopic or photometric redshifts of member galaxies have been obtained was found to consist of two structures at $z \simeq 1.7$ and $z \simeq 2$ [53]. Despite the fact that many of these clumps of emission selected with Planck are not single proto-clusters, they are nevertheless signposts of proto-cluster regions along the line of sight.

An alternative search for candidate high- $z$ proto-clusters has been carried out by Ref. [47, see also Greenslade et al. 2016, in preparation], by cross matching the $857 \mathrm{GHz}$ Planck 
Catalogue of Compact Sources with over $1000 \mathrm{deg}^{2}$ of Herschel survey fields (primarily HATLAS [54] and HerMES [55]) to search for evidence of compact emission from cold clumps of high- $z$ galaxies. Selected at $857 \mathrm{GHz}$, these sources are still "cold", but "warmer" than those selected at $545 \mathrm{GHz}$ by Planck [49], and thus corresponding to a higher dust temperature or redshifts $z \sim 1-2$ for typical dust SEDs. A visual inspection of the Herschel maps at the positions of the Planck compact sources for excesses of red sources identifies a total of 59 proto-cluster candidates. As noted in Ref. [49], few of the Planck high- $z$ sources correspond to a PCCS2 source, so these two alternative methods likely probe different populations of proto-clusters.

Simulations of the distributions of the sub-mm flux from proto-clusters seen by Planck [56, see their figure 7] suggest that the typical 350- $\mu \mathrm{m}$ flux density from a proto-cluster is on the order of $100 \mathrm{mJy}$, below Planck's sensitivity. The detection of such sources in the PCCS2 however indicates a typical $350 \mu \mathrm{m}$ flux density of $\simeq 800 \mathrm{mJy}, 5-10$ times greater than predicted from the simulations. Since the typical highest proto-cluster SFR reached in the simulations was $\simeq 1600 \mathrm{M}_{\odot} \mathrm{yr}^{-1}$, this implies an observed SFR on the order of $\simeq 10^{4} \mathrm{M}_{\odot} \mathrm{yr}^{-1}$, consistent with the observations from blind searches on the Planck maps for cooler protoclusters.

In any case, the samples of "cold" intensity peaks detected by Planck (and in much greater abundance by CORE) are of great interest for the investigation of the evolution of large-scale structure. Follow-up observations of galaxies within them, providing redshift estimates, would be a powerful tool to investigate the early phases of cluster formation, inaccessible by other means. In fact, these overdensities can be detected in the pre-virialization phase, when their intergalactic medium might not have been shock-heated yet, and hence they may not be detectable via X-ray emission or the Sunyaev-Zeldovich (SZ) effect.

The higher resolution of CORE will facilitate a much easier selection of true protoclusters. The search described in Ref. [49] was carried out adopting an angular resolution of $5^{\prime}$, corresponding to a physical size of about $2.5 \mathrm{Mpc}$ at $z=1.5-2$. This resolution is not optimal for detecting proto-clusters. The study by Ref. [57] of 274 clusters with $0.3 \leq z \leq 1.5$ from the Spitzer InfraRed Array Camera (IRAC) Shallow Cluster Survey, using Herschel/SPIRE 250- $\mu \mathrm{m}$ imaging, showed that the density of IR-emitting cluster members dominates over the background field level only within $0.5 \mathrm{Mpc}$ of the cluster centre, while at $r>0.5 \mathrm{Mpc}$ the corrected source density of cluster members is only a small enhancement over the field source density.

A linear scale of $0.5 \mathrm{Mpc}$ corresponds to an angular scale of about $1^{\prime}$ at redshifts in the range 1.5-2.5, i.e. to the angular radius of the CORE beam at $600 \mathrm{GHz}$ for the $1-\mathrm{m}$ telescope option. The $1.5-\mathrm{m}$ option, with its FWHM of $1.6^{\prime}$ at $600 \mathrm{GHz}$ and of $1.2^{\prime}$ at $800 \mathrm{GHz}$, corresponding, in that redshift range, to linear radii of $\simeq 395$ or $\simeq 335 \mathrm{kpc}$, respectively, will be much better suited to detect the bright cluster cores of the kind discovered in Ref. [45] at $z=2.41$ and in Ref. [46] at $z=2.51$ on scales of $\sim 100 \mathrm{kpc}$ (see Fig. 2).

A visual indication of the appearance of such proto-clusters at different resolutions is given in Fig. 3. Though only a single source is identifiable in the Planck maps, both CORE 100 and CORE150 show that the nature of this source is a clear overdensity of fainter sources, as also seen in the Herschel maps. The all-sky nature of CORE would enable us to search for such overdensities over roughly 20 times the area surveyed by Herschel.

The CMB-S4 surveys also have a resolution well suited for proto-cluster detection. However, since most of detectable proto-clusters should be at relatively low redshifts $(z<3)$, their signal is much lower at the longer CMB-S4 wavelengths. Thus these objects are considerably 

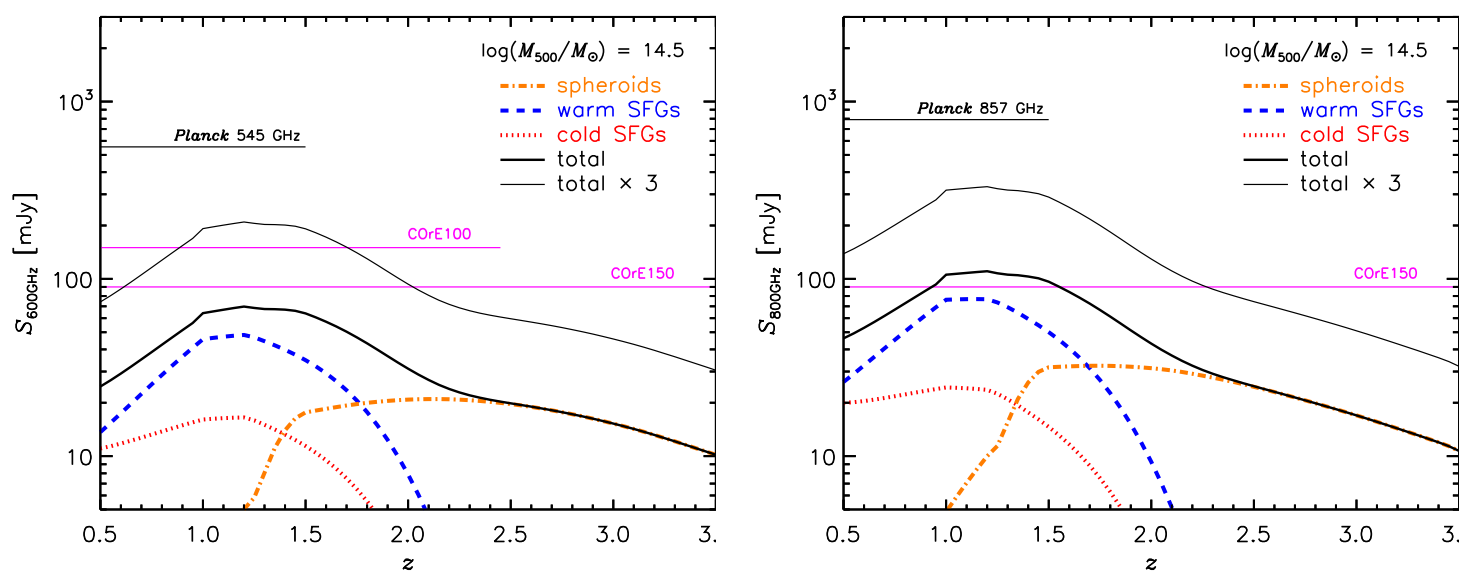

Figure 4. Expected flux density at 600 (left panel) and $800 \mathrm{GHz}$ (right panel) due to dust emission of a cluster with $M_{500}=10^{14.5} \mathrm{M}_{\odot}$ as a function of the cluster redshift. The cluster luminosity includes contributions from normal late-type and starburst galaxies (warm and cold SFGs, respectively) and from proto-spheroidal galaxies (spheroids), computed using the model by [28]. The thick curve shows the average flux density, computed using eq. (4.2). The upper curve is a factor of 3 higher and illustrates the large variance of the cluster IR emission at fixed mass [57, 58]. It must be noted that the Planck and the SPT surveys have detected $z>0.5$ clusters with masses of more that $10^{15} \mathrm{M}_{\odot}$ (see text). The uppermost horizontal line shows to the PCCS2 90\% completeness level in the 'extragalactic zone' for the Planck channel nearest in frequency. The two lower horizontal lines in the left panel show the point source detection limits for the 1-m and 1.5-m telescope options. In the right-hand panel only the detection limit for the 1.5 -m option is shown.

easier to detect at the highest CORE frequencies. As in the case of strongly lensed galaxies, the CMB-S4 data will contribute to determining the SED, hence the photometric redshift (for an assumed dust temperature), the IR luminosity and the SFR of each source.

A complete, unbiased census of these proto-clusters is fundamental to our understanding of the full path of galaxy cluster formation and of the effect of dense environments on galaxy formation and evolution. Such structures, however, are rare and therefore difficult to find. The surface density of proto-clusters serendipitously detectable by CORE (and by CMB-S4) is estimated to be of a few to several $\times 10^{-2} \mathrm{deg}^{-2}[45$, see also Negrello et al. 2016, in preparation]; hence we may expect the detection by CORE of several hundreds to a few thousand violently starbursting proto-cluster cores.

\section{Evolution of the IR emission of galaxy clusters}

The serendipitously detected proto-clusters are likely in different evolutionary stages, from early agglomeration towards the collapse to virial equilibrium. There is, however, also clear evidence of strong evolution in the star-formation activity in virialized clusters [59, and references therein]. The specific SFR increases rapidly from $z \sim 0.2$ to $z \sim 1.3$, mostly driven by the activation of star formation in early-type galaxies at $z \gtrsim 0.9$. CORE will allow us to investigate the evolution of galaxy populations in such clusters, detected via their X-ray emission [60-62] or via the thermal SZ (tSZ) effect [63-65].

The South Pole Telescope (SPT) tSZ survey sample contains clusters with median mass of $M_{500}=3.5 \times 10^{14} \mathrm{M}_{\odot}{ }^{2}$ and covers the redshift range $0.047 \leq z \lesssim 1.7$ [64]. The Planck

\footnotetext{
${ }^{2}$ We define $M_{500}$ as the total mass within the radius for which the mean overdensity is 500 times the mean
} 
survey [65] has been particularly effective at detecting massive $\left(M_{500}>5 \times 10^{14} \mathrm{M}_{\odot}\right.$, and up to $\simeq 2 \times 10^{15} \mathrm{M}_{\odot}$ ) clusters at $z>0.5$ (and up to $z \simeq 1$ ). Using a stacking approach for a sample of 645 clusters whose SZ signal was detected by Planck, Ref. [66] found a significant detection of dust emission from 353 to $857 \mathrm{GHz}$ with an average SED shape similar to that of the Milky Way. Further evidence of dust emission from Planck SZ clusters was provided by the detection of a cross-correlation between the tSZ effect and the cosmic infrared background [CIB; 67].

At $z \lesssim 1$ dense cluster cores are preferentially populated by massive, passively evolving, early-type galaxies. Star-forming galaxies are generally found in the cluster outskirts and in the field. Ref. [57] showed that the star-formation activity in cluster core $(r<0.5 \mathrm{Mpc})$ galaxies from $z=0.3$ to 1.5 is at the field level at $z \gtrsim 1.2$ but is suppressed faster at lower redshifts. A similar conclusion was reached by studying a sample of 57 groups and clusters in the range $0<z<1.6$ using the deepest surveys with Spitzer MIPS (MultiBand Imaging Photometer for Spitzer) and Herschel Photoconductor Array Camera and Spectrometer (PACS) and SPIRE, on blank and cluster fields [68].

The ratio of the mean IR luminosity of galaxies in clusters (within $1 \mathrm{Mpc}$ from the cluster centre) to that of galaxies in the field, for $z<1.2$, was found to be [57]

$$
f(z)=\frac{L_{\mathrm{IR}, \text { cluster }}}{L_{\mathrm{IR}, \text { field }}} \simeq 5.77 e^{-0.34 t_{\mathrm{Gyr}}}
$$

with large uncertainties; here $t_{\mathrm{Gyr}}$ is the cosmic time in Gyr. The cluster IR luminosity then can be written

$$
L_{\mathrm{IR}, \text { cluster,tot }}(z)=\Psi_{\mathrm{IR}}(z) f(z) \delta V_{\text {comoving }}=\Psi_{\mathrm{IR}}(z) f(z) M_{\text {cluster }} /\left\langle\rho_{0}\right\rangle,
$$

where $\Psi_{\mathrm{IR}}(z)$ is the IR comoving luminosity density, $\delta$ is the cluster density contrast, $\rho_{\text {cluster }} /\langle\rho\rangle$, $\langle\rho\rangle$ is the mean matter density whose present day value is $\left\langle\rho_{0}\right\rangle=2.7755 \times 10^{11} h^{2} \Omega_{\mathrm{m}} \mathrm{M}_{\odot} \mathrm{Mpc}^{-3}$, $V_{\text {comoving }}$ is the cluster volume and $M_{\text {cluster }}$ is the cluster mass.

According to figure 17 of Ref. [69], $\Psi_{\mathrm{IR}}(z) \propto(1+z)^{3}$ and for the standard $\Lambda$ CDM cosmology we have, approximately, $f(z) \propto(1+z)^{3.8}$ for $z \lesssim 1$, so that the cluster IR luminosity at fixed mass evolves very strongly $\left[L_{\mathrm{IR} \text {,cluster,tot }}(z) \propto(1+z)^{6.8}\right.$, up to $\left.z \sim 1\right]$.

Ref. [58] presented a detailed study of star formation in 11 near-IR selected, spectroscopically confirmed, massive $\left(M \gtrsim 10^{14} \mathrm{M}_{\odot}\right)$ clusters at $1<z<1.75$. They found that the star formation in cluster galaxies at $z \gtrsim 1.4$ is largely consistent with field galaxies at similar epochs, confirming earlier results from the Spitzer/IRAC Shallow Cluster Survey [70]. Although the samples were uniformly selected there are quite strong variations in star formation from cluster to cluster.

We have used these results to make tentative predictions of expected flux densities of clusters as a whole. Figure 4 shows the expected flux densities due to dust emission from a cluster with mass $M_{500}=10^{14.5} \mathrm{M}_{\odot}$ as a function of redshift. The cluster luminosities were obtained using eq. (4.2), and the contributions of late-type, starburst and proto-spheroidal galaxies were computed using the model from Ref. [28].

As shown in Fig. 4 we expect that at $800 \mathrm{GHz}$ the CORE150 version will detect the dust emission of the brightest $M_{500} \simeq 10^{14} \mathrm{M}_{\odot}$ clusters and of typical $M_{500} \simeq 10^{14.5} \mathrm{M}_{\odot}$ clusters at $1 \lesssim z \lesssim 1.5$. More massive clusters will be detected over broader redshift ranges. Stacking

matter density of the Universe. Note that frequently $M_{500}$ is defined to correspond to an overdensity 500 times the critical density. 
will allow us to carry out a statistical investigation of the evolution of the cluster IR emission over a much broader redshift baseline than has been possible so far. Targets for stacking will abound. By the time CORE will fly, eROSITA (extended ROentgen Survey with an Imaging Telescope Array), expected to be launched in 2017, should have provided an all-sky deep Xray survey detecting $\sim 10^{5}$ galaxy clusters out to $z>1$ [61]. About 38,000 cluster detections out to $z \gtrsim 2$ are expected, via the tSZ effect, from the baseline CORE mission, and about 50,000 with CORE150; combining CORE150 and CMB-S4 data it will be possible to detect around 200,000 clusters in the sky visible from the South Pole (Melin et al., in preparation).

For comparison, Herschel has allowed the study of the IR emission from clusters up to $z \simeq 1.7$ [58], but the sample comprises only 11 clusters. The Herschel data have shown large variations in cluster properties, highlighting the need for evolutionary studies of large, uniform cluster samples over a broad redshift range. CORE will fulfill this need.

The situation is somewhat less favourable at $600 \mathrm{GHz}$, where, however, CORE will nevertheless substantially improve over Planck. At this frequency a direct source detection by CORE will be possible only for cluster masses $M_{500} \gtrsim 10^{14.5} \mathrm{M}_{\odot}$. But again, the stacking approach will allow substantial progress in the exploration of the evolution of the IR emission as a function of cluster mass and redshift. This will shed light on the mechanisms which drive the evolution of massive spheroidal galaxies in clusters, from actively star forming to passively evolving in dense environments, and on the role of nuclear activity in this process.

Figure 4 shows that, according to the model in Ref. [28], different galaxy populations are expected to dominate the cluster sub-mm emission at different redshifts. At $z \lesssim 0.5$ the main contribution comes from late-type galaxies with relatively cold dust temperatures. This is consistent with the dust temperature, $T_{\mathrm{d}} \simeq 24 \mathrm{~K}$, estimated in Ref. [66] for their sample of clusters detected by Planck, with an estimated mean redshift $z=0.26 \pm 0.17$. This study also found a slight increase of dust temperature with redshift, comparing clusters at $z$ larger and smaller than 0.25 . Such an increase is indeed predicted by the model, due to the increasing contribution of the warmer starburst population that takes over above $z \simeq 0.5$ and out to $z \simeq 1.7$. At still higher redshifts the dominant population is the proto-spheroidal galaxies in the process of forming most of their stars.

Once more, we will take advantage of the synergies with CMB-S4, as pointed out in the previous sections. The characterization of the IR emission in individual clusters, obtained combining CORE and CMB-S4 data, is essential to extract the relativistic correction in the hottest clusters and the contribution from non-thermal electrons to the SZ effect [71, 72]. Relativistic corrections would allow us to estimate in an independent way the plasma temperature while non-thermal contributions provide information on outflows from star formation and/or from nuclear activity.

\section{The Cosmic Infrared Background (CIB)}

Progress in our understanding of the power spectrum of the CIB that will be made possible by CORE has been discussed in Ref. [1]. Briefly, the better CORE angular resolution, compared to Planck, will allow us to measure, in a uniform way, the CIB power spectrum over an unprecedented range of frequencies and of angular scales (from $\sim 1 \operatorname{arcmin}$ to tens of degrees), thus breaking the degeneracy between the Poisson contribution and that of non-linear effects (one-halo term), that complicates the interpretation of Planck measurements without resorting to external data. Accurate determinations of the CIB power spectrum at different 
frequencies provide, on the one hand constraints on the evolution of the cosmic star-formation density and, on the other hand, on halo masses associated with sources of the CIB [73-75].

The exploitation of CIB anisotropies as a probe of star-formation history in the Universe and also as a dark matter tracer requires a careful removal of foreground radiations, the most important of which, at sub-mm wavelengths, is the Galactic thermal dust emission. The separation of dust and CIB components is challenging since both have frequency spectra scaling approximately as modified black-bodies with similar emissivity indices. An approach successfully applied to Planck temperature maps combines the frequency information, to reconstruct the Galactic thermal dust model, with spatial information, taking advantage of different power spectra of the components [76]. In fact, the CIB power spectrum scales approximately as $\ell^{-1}$ [73], while the Galactic dust power spectrum scales approximately as $\ell^{-2.7}$ or $\ell^{-2.8}$ for $\ell>110$ [74]. With its much more numerous frequency channels, higher sensitivity and better angular resolution, CORE will substantially improve over Planck both in the spectral and spatial characterization, thus enabling a much easier and more robust separation of the two components. Obviously, a better Galactic thermal dust model will also allow an improved cleaning of CMB maps.

Moreover, the large number of CORE channels will make it possible to investigate in detail the decorrelation of power spectra measured in different frequency bands. A decorrelation is expected because the redshift distribution of CIB sources shifts to higher and higher redshifts with decreasing frequency. Accurate measurements of the CIB cross-spectra for different frequency channels set strong constraints on the frequency dependence of redshift distributions, hence on the evolution of the cosmic SFR. Ref. [77] found that the CORE design is very close to the optimal design for improving the SFR constraints at all redshifts.

As pointed out by Ref. [78], the angular power spectrum of the CIB is a sensitive probe of primordial non-Gaussianity. It potentially outperforms the forthcoming large-scale galaxy redshift surveys because the CIB traces the large-scale structure over a much larger comoving volume. Again, the key factor is an accurate subtraction of the Galactic thermal dust emission, achievable with the numerous sub-mm channels of CORE. This will make possible to constrain the "local type" primordial non-Gaussianity parameter called $f_{\text {nl }}$ down to an amplitude $\left|f_{\text {nl }}\right|<1$.

Another interesting issue is the possibility of exploiting the multi-frequency measurements of the dipole spectrum to constrain the CIB intensity spectrum [79-82]. This arises from the fact that the dipole amplitude is directly proportional to the first derivative of the photon occupation number. High accuracy measurements of the CIB dipole amplitude at sub-mm wavelengths will provide important constraints on the CIB intensity, currently known with $\mathrm{a} \simeq 30 \%$ uncertainty [83]. Such a large uncertainty constitutes a major limitation to our understanding of the dust-obscured star formation phase of galaxy evolution. The exploitation of this possibility requires a very accurate inter-channel cross-calibration and a careful control of the systematics from the foreground Galaxy subtraction [84]. A detailed discussion of this topic will be presented in a companion paper (Burigana et al., in preparation).

\section{Resonant scattering on metals and ions at high redshifts}

The multi-frequency CORE observations will not only allow us to separate the usual foreground signals, such as the diffuse Galactic emission and point sources, but might also enable the identification of subtler astrophysical signals. In particular, metals and ions in the inter- 


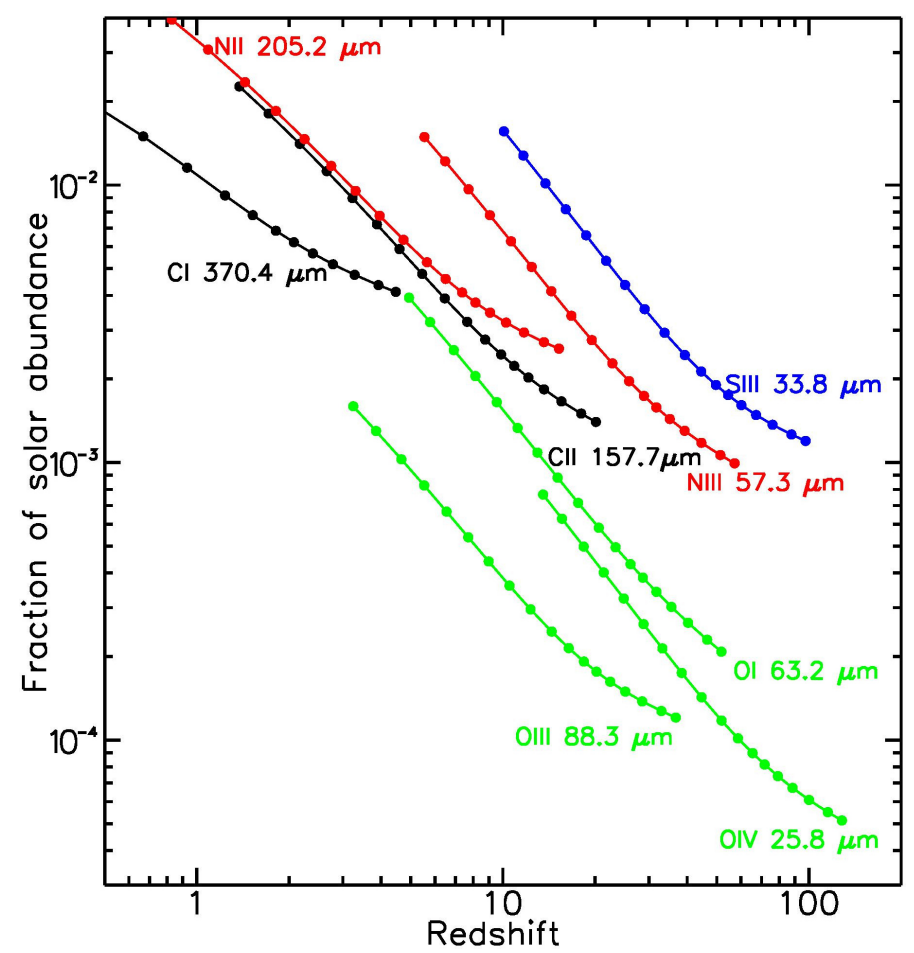

Figure 5. Constraints on the abundance of several metal and ion species as a function of redshift, obtained by comparing the power spectra measured in different CORE channels with that measured at $80 \mathrm{GHz}$. This assumes an inter-channel calibration accurate at the level of $0.001 \%$, and that the uncertainties in the beam characterization can be neglected.

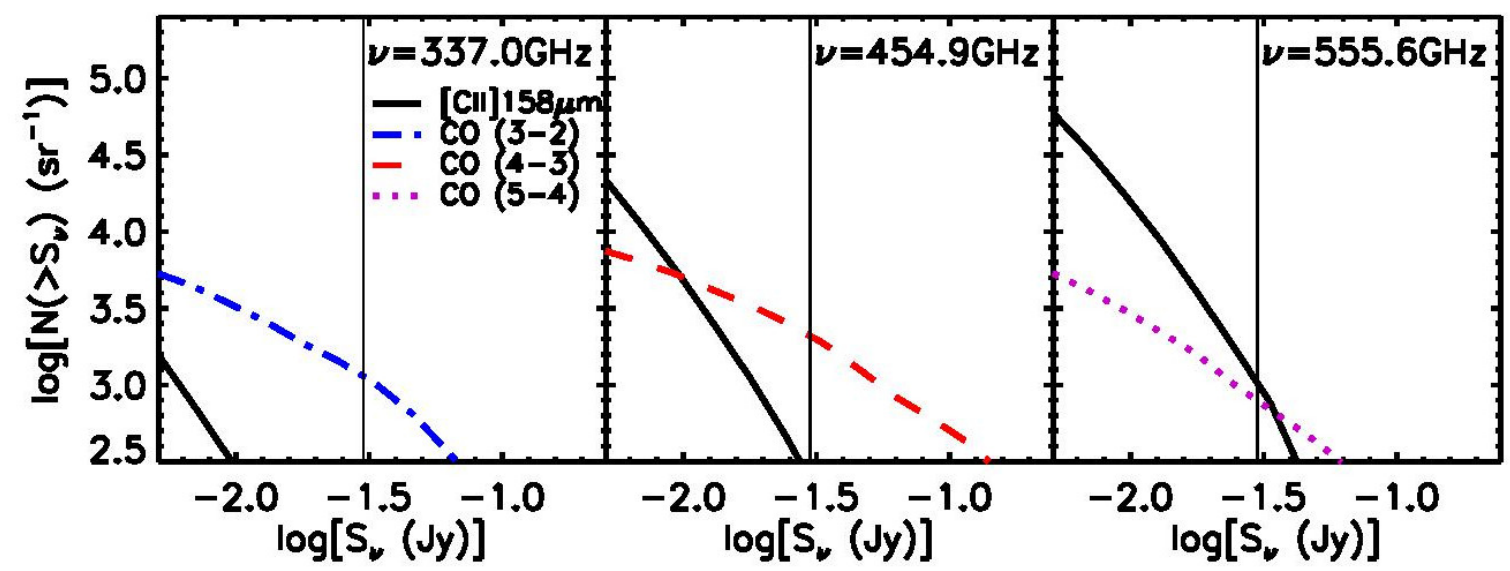

Figure 6. Integral counts of $\mathrm{CO}$ and $\mathrm{C}+$ lines at three frequencies within the CORE range with a resolution of $R=20$. The vertical solid lines correspond to a detection limit of $30 \mathrm{mJy}$.

galactic medium (IGM) leave signatures in the anisotropy pattern of the CMB intensity and polarization.

As shown in Refs. [85] and [86], in low over-density regions the dominant mechanism coupling the CMB photons with metals and ions is resonant scattering. On large angular scales such scattering blurs the intrinsic anisotropy pattern and induces a frequency dependent 
variation of the observed angular power spectrum given by

$$
\delta C_{l}^{j i} \equiv C_{l}^{j}-C_{l}^{i} \approx-2 \delta \tau_{M}^{j i} C_{l}^{\mathrm{intr}}=-2\left(\tau_{M}^{j}-\tau_{M}^{i}\right) C_{l}^{\mathrm{intr}},
$$

where $C_{l}^{\text {intr }}$ is the intrinsic CMB angular power spectrum and $\delta \tau_{M}^{j i}$ is the increment of the optical depth associated with resonant transitions of one or several species corresponding to two different observing frequencies $\nu_{i}$ and $\nu_{j}$.

Low frequency channels are affected by resonant lines produced at high redshifts. But at high enough redshifts the abundance of metals and ions is negligible; hence the CMB power spectrum measured at low frequencies is the intrinsic one, so that low frequency channels can be used as a reference, against which to compare observations at higher frequencies that correspond to lower redshift lines. This comparison allows us to set constraints on the abundance of different species of metals and ions at different cosmological epochs [see 85, 86, for more details].

However, real life is significantly more complicated. Besides foregrounds there are other issues, the most important being inter-channel calibration and beam characterization [87]. In the future, the proposed PIXIE experiment [88] could provide absolutely calibrated lowresolution maps of the sky to improve the channel inter-calibration and mitigate this problem. If we assume that foregrounds can be projected out down to negligible levels and that we can achieve an inter-channel calibration at the $0.001 \%$ level (approximately 100 times better than for the Planck-HFI cosmological channels), then a comparison of the power spectrum measured by CORE at $80 \mathrm{GHz}$ with those measured at higher frequencies would yield the constraints (at $3 \sigma$ or $>99.7 \%$ confidence level) on different species shown in Fig. 5. These limits are obtained assuming that only one transition contributes to the signal, and that the beams can be characterized with arbitrary precision. For a given species, constraints are tighter at higher redshifts/lower frequencies, where the control of free-free and synchrotron emission is more demanding.

High sensitivity, multi-frequency CMB observations are also sensitive to other signals involving atoms and molecules, such as the collisional/rotational emission of $\mathrm{CO}$ and the $\mathrm{C}+157.7 \mu \mathrm{m}$ line $[82,89,90]$ or the Field-Wouthuysen effect on OI atoms [91, 92]. The poor spectral resolution of CMB experiments implies a strong dilution of the lines, making them undetectable in individual sources. But an improvement of the spectral resolution, $R=\nu / \Delta \nu$, by a factor of several, at least for some high frequency channels, would make possible the detection of thousands of sources in these lines. Examples are shown in Fig. 6 for a modest $R=20$.

The integral counts shown in this figure were worked out exploiting the model in Ref. [28] for the cosmological evolution of the IR luminosity function, coupled with relations between line intensities and IR luminosities. For the CO lines we have used the relations of Ref. [93] and for the $\mathrm{C}+157.7 \mu \mathrm{m}$ line that by Ref. [94]. More details on the calculations can be found in Ref. [82]. As illustrated by Fig. 6, an all-sky survey with $R=20$ is expected to detect line emission brighter than 30 mJy (a flux density limit achievable by CORE150) of $\sim 10^{4}$ galaxies in the "extragalactic zone". The $\mathrm{C}+157.7 \mu \mathrm{m}$ line becomes increasingly important with increasing frequency.

\subsection{Radio sources}

The overwhelming majority of extragalactic radio sources detectable by CORE are blazars, i.e. sources whose radio emission is dominated by Doppler-boosted emission from relativistic 
jets closely aligned with the line of sight [95]. These objects, with their extreme properties, are of special interest since they are also strong $\gamma$-ray sources. About $90 \%$ of the firmly identified extragalactic sources and about $94 \%$ of the "associated" sources (i.e. of sources having a counterpart with a $>80 \%$ probability of being the real identification) in the Third Fermi Large Area Telescope (LAT) catalogue [96] are blazars.

Accurate source counts over large flux density intervals provide key constraints on blazar evolutionary models. Because high frequency surveys are still far less extensive than those at low frequencies, evolutionary models for blazar populations, flat spectrum radio quasars (FSRQs) and BL Lacertae sources (BL Lacs), are less developed than those for steep-spectrum radio sources $[97,98]$. For example, while clear evidence for downsizing ${ }^{3}$ was reported in the case of steep-spectrum sources [98, 100], the available data are insufficient to test if this is also the case for FSRQs; for BL Lacs the constraints on evolutionary parameters are even weaker. This situation hampers sharp tests of unified models of radio sources.

Furthermore, source counts constrain the compactness of the synchrotron-emitting regions in the jets. Already the counts at mm wavelengths available in 2011 allowed Ref. [30] to rule out simple power-law spectra for these sources and to constrain models for spectral breaks of the synchrotron emission in blazar jets. Furthermore, the assumption of uniform spectral properties for the whole blazar population was found to be, at best, only marginally consistent with the data. A clearly better match with the observed counts was obtained by assuming that BL Lacs have, on average, substantially higher break frequencies than FSRQs, suggesting that the synchrotron emission comes from more compact regions. This led to the prediction of a substantial increase of the BL Lac fraction at still higher frequencies and at bright flux densities. To test this prediction surveys at sub-mm wavelengths, such as those carried out by CORE, are paramount.

In order to characterize the blazar synchrotron peak it is crucial to push the photometry to sub-mm wavelengths, which will be provided by CORE. Ref. [101] investigated the global spectral energy distributions (SEDs) of a sample of 104 extragalactic radio sources drawn from the Planck Early Release Compact Source Catalogue (ERCSC), combining Planck data with simultaneous observations ranging from radio to $\gamma$-rays. They have shown that Planck data provide key information on the energy spectrum of relativistic electrons responsible for the synchrotron emission, with implications for the acceleration mechanisms provided by shocks along the relativistic jets.

The further analysis in Ref. [102] has confirmed that the flattest high-frequency radio spectral indices are close to zero, indicating that the original energy spectrum of accelerated electrons is much harder than commonly thought, with power-law index around 1.5 instead of the canonical 2.5. The radio spectra of these sources peak at remarkably high frequencies, tens of $\mathrm{GHz}$, and there are significant statistical differences between subclasses of Active Galactic Nuclei (AGN) at high frequencies, particularly between highly-polarized quasars and BL Lac objects.

An interesting open question is the geometry of the emitting region. The data analysed in Ref. [103] are consistent with a single synchrotron emission zone, but their analysis was limited to frequencies up to $217 \mathrm{GHz}$. However, simultaneous SED data, including Planck photometry, for some particularly bright sources suggest double emission zones peaking at different frequencies with a transition occurring at sub-mm/far-IR wavelengths [104, and references therein]. As illustrated by Fig. 7 and by Table 2, the deeper sub-mm surveys

\footnotetext{
3"Downsizing" refers to the very different evolutionary behaviour of high- and low-luminosity sources, in the sense that the redshift of the peak space density of sources decreases with luminosity [99].
} 
Table 2. Number of Planck/PCCS2 counterparts to sources in the PACO-bright sample, compared with the number of expected CORE detections for the 1-m and 1.5-m telescope options.

\begin{tabular}{lrrrrrr}
\hline \hline Frequency $[\mathrm{GHz}]$ & \multicolumn{1}{c}{70} & 100 & 143 & 217 & 353 & \multicolumn{1}{c}{545} \\
\hline PCCS2 & $103(71 \%)$ & $132(91 \%)$ & $135(93 \%)$ & $91(63 \%)$ & $3(2 \%)$ & $1(0.7 \%)$ \\
\hline CORE100 & $143(99 \%)$ & $142(98 \%)$ & $141(97 \%)$ & $141(97 \%)$ & $137(95 \%)$ & $91(63 \%)$ \\
\hline CORE150 & $145(100 \%)$ & $145(100 \%)$ & $145(100 \%)$ & $145(100 \%)$ & $142(98 \%)$ & $120(83 \%)$ \\
\hline \hline
\end{tabular}

by CORE will increase by about two orders of magnitude the number of sources detected up to $600 \mathrm{GHz}$. The improved constraints on the spectral energy distributions at sub-mm wavelengths entail correspondingly better constraints on the geometry of the emitting regions.

Key information on the flow of the plasma within the relativistic jets is provided by variability. Signatures of evolving shocks in the strongest radio flares were seen in a study of Planck sources [102], although much of the high frequency variability may be better approximated by achromatic variations. These results are compatible with the standard shocked jet model, but other interpretations are also possible. CORE will shed light on this by providing much larger samples and a broader spectral coverage.

Definite conclusions on all the above issues are currently hampered by the limited statistics. To illustrate the improvement made possible by CORE with respect to Planck we have considered the complete Planck-ATCA Co-eval Observations (PACO) "bright" sample [103]. The sample comprises the 189 sources with AT20G flux densities $S_{20 \mathrm{GHz}}>500 \mathrm{mJy}$, only a few of which were detected by Planck beyond $217 \mathrm{GHz}$ (see Table 2). Figure 7 shows the continuum spectra of the subset of 145 sources for which we could obtain good fits with smooth functions that can be used to extrapolate the spectra to higher frequencies. ${ }^{4}$ Using such fits we find that the fraction of these sources detectable by CORE is $\geq 95 \%$ for CORE100, and $\simeq 100 \%$ for CORE150, up to $353 \mathrm{GHz}$ (cf. Fig. 7 and Table 2)

Together with CORE, CMB-S4 will extend by a factor of 30 the frequency range over which accurate number counts of radio sources down to tens to hundred mJy will be determined. At the moment, counts down to at least these levels are available only at $\nu \leq 20 \mathrm{GHz}$ [see Ref. 105, for a review], and between 95 and $220 \mathrm{GHz}$, where the Planck counts are supplemented at fainter flux densities by those from the South Pole Telescope [SPT; 106] and from the Atacama Cosmology Telescope [ACT; 107]. At other frequencies $\nu \geq 30 \mathrm{GHz}$ the best current information on radio source counts comes from Planck [108] and is limited to flux densities not much below $1 \mathrm{Jy}$.

The characterization of the blazar emission at both $\mathrm{mm}$ and sub-mm wavelengths is an essential ingredient for understanding the physics of these sources [101, 109, 110]. There is an obvious synergy, in this respect, between CMB-S4 and CORE surveys. The synergy extends also to the study of variability properties. Multi-epoch (sub-)millimetre observations can serendipitously detect variable objects and flares in BL Lacs and FSRQs, particularly in regions with high scan coverage, as seen by Planck in the blazar S5 1803+78 [111]. Planck/HFI carried out five full surveys of the sky, while CORE is expected to make six to ten full surveys. In combination with CMB-S4 observations, CORE will enable a multifrequency study of variability on scales from months to several years.

\footnotetext{
${ }^{4}$ We have updated the analysis of Ref. [103] using the PCCS2 data. The number of sources with smooth spectra decreased from 147 to 145, with no significant change to the earlier conclusions.
} 


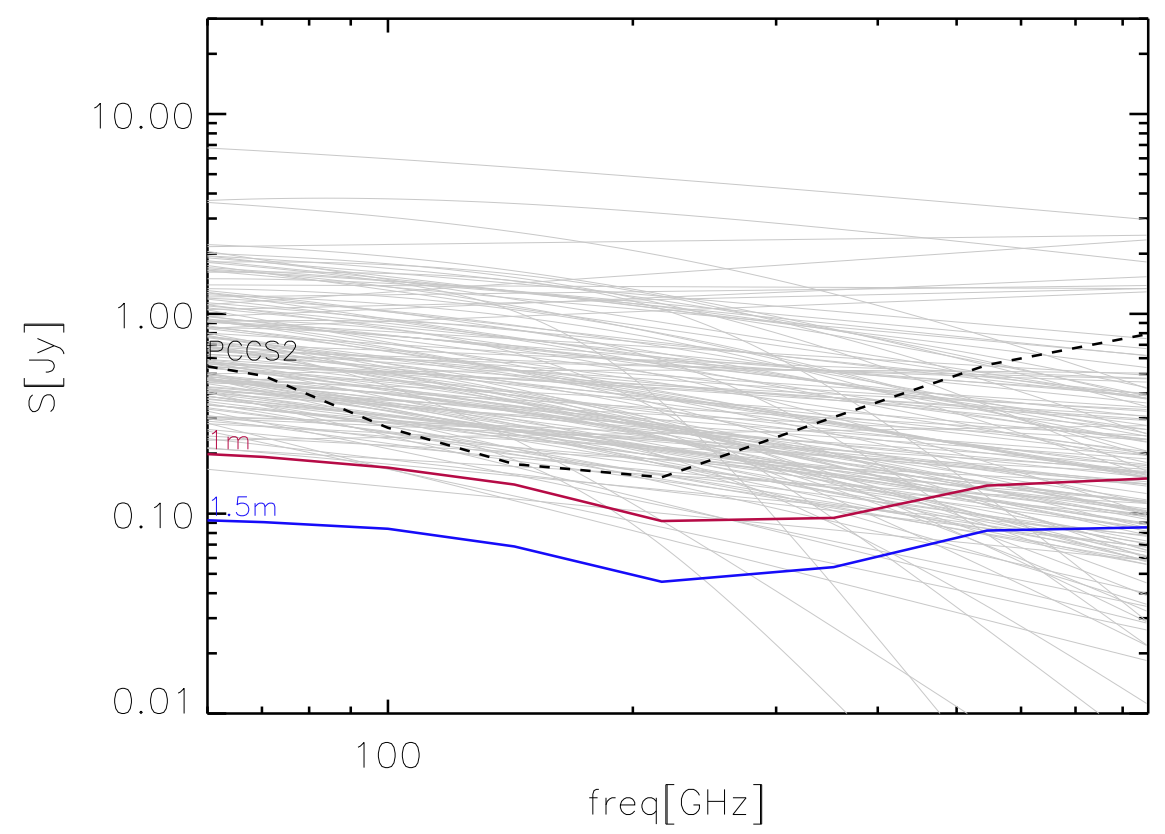

Figure 7. Continuum spectra of PACO-bright sources compared with the $90 \%$ completeness limits of the Planck/PCCS2 catalogue (dashed black line) and with the $4 \sigma$ detection limits for the CORE 1-m (CORE100) and 1.5-m (CORE150) telescope sizes (solid red and blue lines, respectively). Note that, particularly at the highest frequencies, the fraction of PACO-bright sources whose fitted spectra are above the Planck/PCCS2 90\% completeness limits is substantially larger than the number of actual Planck/PCCS2 detections, listed in Table 2; this may suggest that the fits over-predict the flux densities at frequencies beyond the highest frequency detections or that the PCCS2 completeness level is somewhat overestimated.

Table 3. Simulations of extragalactic sources in polarization: technical specifications

\begin{tabular}{|c|c|c|c|c|c|c|c|c|}
\hline \multirow[b]{2}{*}{$\begin{array}{l}\text { Freq. } \\
{[\mathrm{GHz}]}\end{array}$} & \multicolumn{4}{|c|}{ CORE100 (1-m telescope) } & \multicolumn{4}{|c|}{ CORE150 (1.5-m telescope) } \\
\hline & $\begin{array}{l}\text { FWHM } \\
\text { [arcmin] }\end{array}$ & $\begin{array}{l}\text { Noise } \\
{[\mathrm{mJy}]}\end{array}$ & $\begin{array}{c}\text { Pix. size } \\
\text { [arcsec] }\end{array}$ & Pixel no. & $\begin{array}{l}\text { FWHM } \\
\text { [arcmin] }\end{array}$ & $\begin{array}{l}\text { Noise } \\
{[\mathrm{mJy}]}\end{array}$ & $\begin{array}{c}\text { Pix. size } \\
\text { [arcsec] }\end{array}$ & Pixel no. \\
\hline 60 & 21.0 & 3.26 & 175 & $512 \times 512$ & 14.0 & 1.54 & 110 & $512 \times 512$ \\
\hline 100 & 12.6 & 1.88 & 105 & $1024 \times 1024$ & 8.4 & 0.52 & 70 & $1024 \times 1024$ \\
\hline 145 & 8.7 & 0.98 & 70 & $1024 \times 1024$ & 5.8 & 0.40 & 50 & $1024 \times 1024$ \\
\hline 220 & 5.7 & 1.18 & 45 & $1024 \times 1024$ & 3.8 & 0.52 & 30 & $2048 \times 2048$ \\
\hline 340 & 3.7 & 3.10 & 30 & $2048 \times 2048$ & 2.5 & 1.06 & 20 & $2048 \times 2048$ \\
\hline 450 & 2.8 & 5.30 & 20 & $2048 \times 2048$ & 1.9 & 1.66 & 10 & $2048 \times 2048$ \\
\hline 600 & 2.1 & 6.70 & 20 & $2048 \times 2048$ & 1.4 & 2.84 & 10 & $2048 \times 2048$ \\
\hline 800 & $\ldots$ & $\ldots$ & $\ldots$ & $\ldots$ & 1.1 & 4.04 & 10 & $2048 \times 2048$ \\
\hline
\end{tabular}

\section{Detecting sources in polarization}

The preliminary estimates [1] suggested that CORE will allow a real breakthrough in the characterization of the polarization properties of extragalactic sources. However, those estimates were only tentative, being based on simplifying assumptions that allowed analytical 


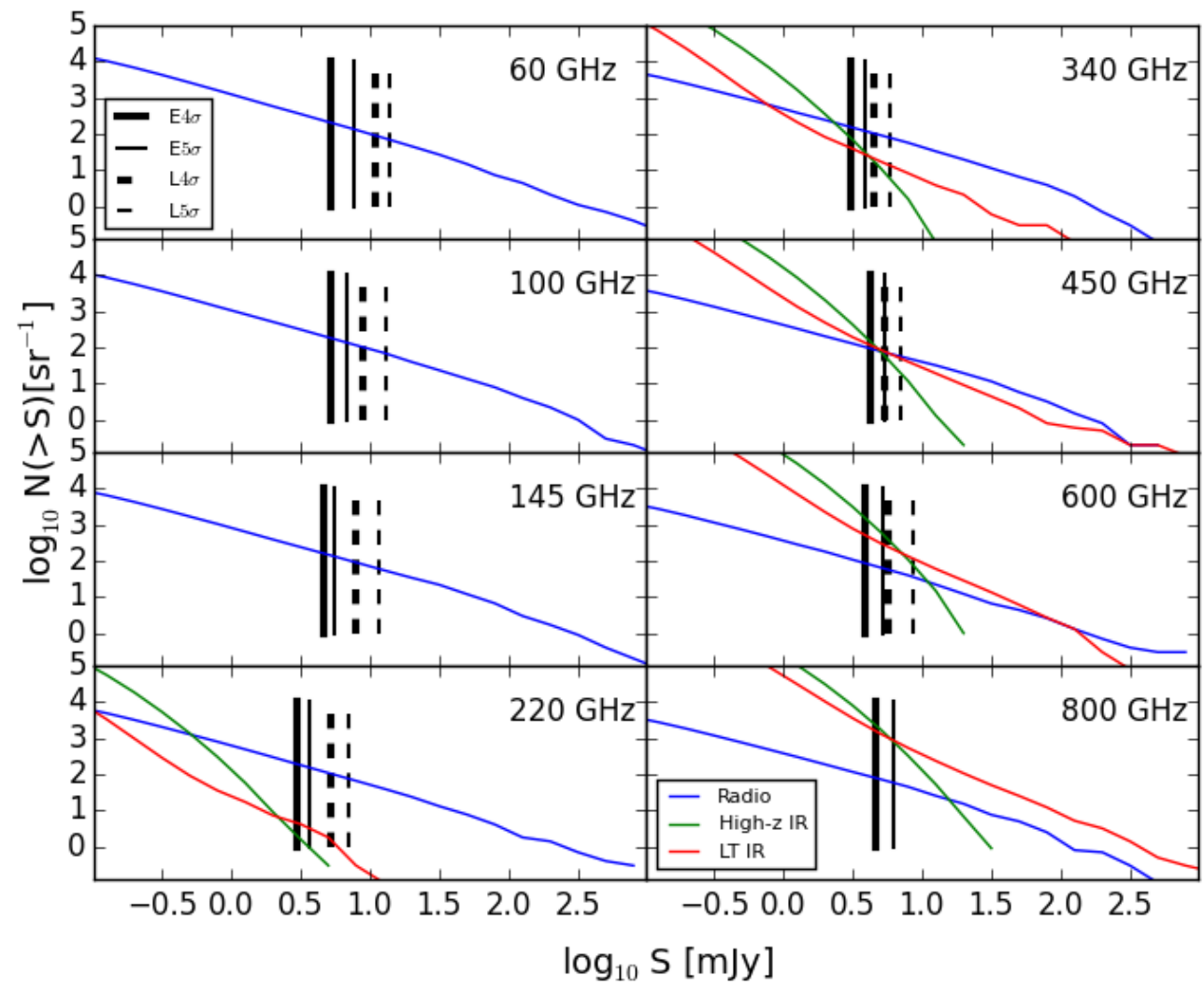

Figure 8. Comparison of the estimated source number counts in polarization for a selection of CORE channels and different source populations: radio sources (solid blue line); and two populations of dusty galaxies (proto-spheroids and late-type, spiral and starburst, galaxies). Proto-spheroids, labelled "High-z IR" (solid green line) dominate at faint flux densities while late-types (LT IR, solid red lines) dominate at the brighter flux densities. The vertical lines show the $4 \sigma$ and $5 \sigma$ detection limits obtained from the simulations for the 1-m (dashed) and 1.5-m (solid) telescope (see Tables 4 and 5 for more details).

calculations. We have now re-assessed them by means of realistic simulations in polarization (the simulations described in Ref. [1] were only in total intensity), carried out for the two options for the telescope size.

The relevant technical details of the simulations are summarized in Table 3. The size of the simulated patch varies with frequency, on account of the varying angular resolution of the survey, quantified by the FWHM of the telescope beam. In the 1-m telescope case, the patch size decreases from $619 \mathrm{deg}^{2}$ at $60 \mathrm{GHz}$ to $129 \mathrm{deg}^{2}$ at $600 \mathrm{GHz}$; in the $1.5-\mathrm{m}$ case it decreases from $245 \mathrm{deg}^{2}$ at $60 \mathrm{GHz}$ to $33 \mathrm{deg}^{2}$ at $800 \mathrm{GHz}$. Given the smallness of the patches, a flat-sky approximation was used.

Our simulations include both radio sources and star-forming galaxies. The latter comprise two sub-populations, late-type galaxies and what we are referring to as proto-spheroids, which have different clustering properties [e.g. 28, 112]. We started from source counts using models that accurately reproduce observational data, specifically the model in Ref. [30] for radio sources and the model in Ref. [28] for star-forming galaxies. 


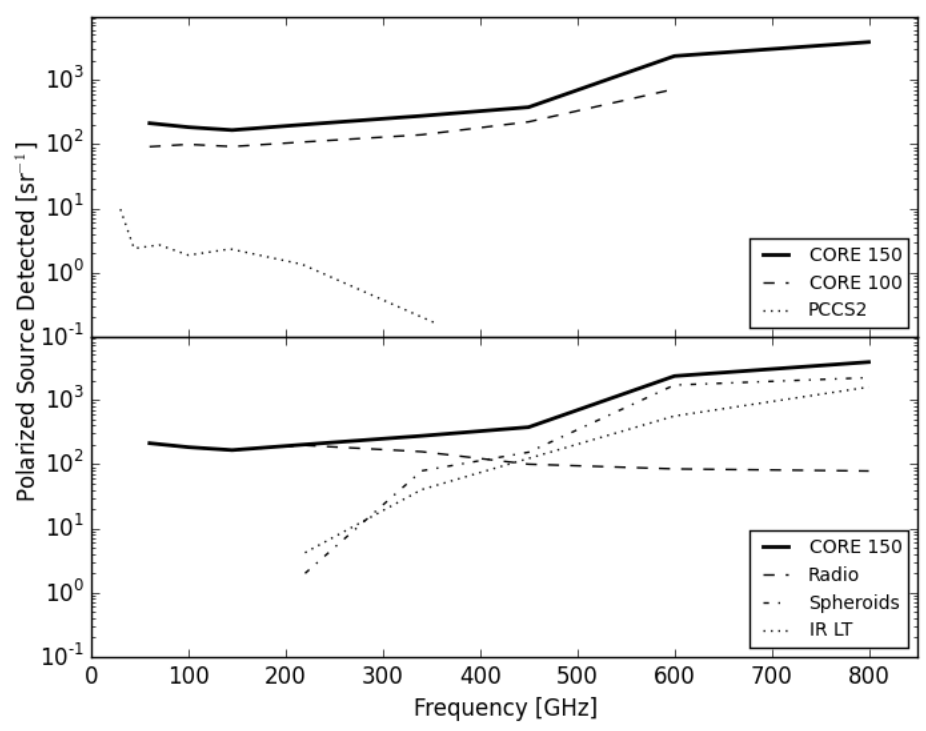

Figure 9. Upper panel: predicted numbers of extragalactic sources detected in polarized flux density as a function of frequency for the two CORE configurations (solid and dashed lines) compared to the numbers of sources detected in polarization by Planck (PCCS2, dotted line). Lower panel: contributions of the different source populations to the counts in polarized flux density as a function of frequency for the CORE150 configuration. The dashed, dot-dashed and dotted lines refer to radio sources ("radio"), proto-spheroidal galaxies ("spheroids") and late-type galaxies "IR LT"), respectively, while the thick solid line shows the total.

We have selected the sets of frequencies, specified in Table 3, in the range covered by CORE and for each frequency we populated sky patches with sources in the flux density range $0.01 \mathrm{mJy}-100 \mathrm{Jy}$, assigning to each source a flux density drawn at random from the number counts. For each simulation we checked the consistency, within Poisson errors, of the counts of simulated sources with those given by the model. Then we associated with each source a polarization degree randomly extracted from probability distributions appropriate for each source population, as detailed below. The polarization angles were drawn at random from a uniform probability distribution.

The most extensive study of the polarization properties of extragalactic radio sources at high radio frequencies was carried out in Ref. [113]. These authors obtained polarization data for 180 extragalactic sources extracted from the Australia Telescope 20-GHz (AT20G) survey catalogue and observed with the Australia Telescope Compact Array (ATCA) during a dedicated, high-sensitivity run $\left(\sigma_{\mathrm{p}} \simeq 1 \mathrm{mJy}\right)$. Complementing their data with polarization information for seven extended sources from the 9-yr Wilkinson Microwave Anisotropy Probe (WMAP) co-added maps at $23 \mathrm{GHz}$, they obtained a roughly $99 \%$ complete sample of extragalactic sources brighter than $S_{20 \mathrm{GHz}}=500 \mathrm{mJy}$ at the selection epoch.

The sample has a $91.4 \%$ detection rate in polarization at $20 \mathrm{GHz}$. The results are in general agreement with those of other polarization surveys at high radio frequencies, carefully reviewed in Ref. [114] and, most recently, in Ref. [115]. The distribution of polarization degrees was found to be well described by a log-normal function with mean and dispersion of $2.14 \%$ and $0.90 \%$, respectively. We have assumed that this distribution holds at all the frequencies 
considered, consistent with the results of Ref. [116].

Most recently, Ref. [117] applied the stacking technique to estimate the average fractional polarization from 30 to $353 \mathrm{GHz}$ of a sample of 1560 compact sources - essentially all radio sources - detected in the $30 \mathrm{GHz}$ Planck all-sky map. The average and median polarization fractions for the 881 sources, presumably extragalactic, outside the Galactic mask were found to be $\simeq 3 \%$ and $\simeq 1.9 \%$, approximately independent of frequency.

In the case of star-forming galaxies, the polarized emission in the CORE frequency range is dominated by dust, except perhaps at the lowest frequencies, where the polarization due to synchrotron emission may take over, at least for low- $z$ galaxies. But at low frequencies the polarized emission of these galaxies is undetectable by CORE in any case.

Polarization properties of dusty galaxies as a whole are almost completely unexplored. The only available information has come from SCUPOL, the polarimeter for SCUBA on the James Clerk Maxwell Telescope, that has provided polarization measurements at $850 \mu \mathrm{m}$ for only two galaxies, M 82 [118] and M 87 [119]. However the global polarization degree has been published only for $\mathrm{M} 82$ and is $\Pi=0.4 \%$. This low value is in keeping with the notion that the polarized dust emission integrated over the whole galaxy is reduced because of the complex structure of galactic magnetic fields, with reversals along the line of sight, and also of the disordered alignment of dust grains. For our simulations we adopted a log-normal probability distribution of $\Pi$ with mean of $0.5 \%$ and dispersion of $1.0 \%$.

Integrating the Planck dust polarization maps over a $20^{\circ}$ wide band centred on the Galactic plane we find an average value of the Stokes $Q$ parameter of about $2.7 \%$. We may then expect a similar value for spiral galaxies seen edge-on. For a galaxy seen with an inclination angle $\theta$ we expect that the polarization degree is reduced by a factor $\cos (\theta)$. If all galaxies are about as polarized as ours, the average polarization fraction for unresolved galaxies, averaged over all possible orientations, should be about half of $2.7 \%$, i.e. around $1.4 \%$. If so our choice of the mean polarisation degree would be quite conservative and the number of detections in polarisation of dusty galaxies would be underestimated by a factor of around 4 .

The angular clustering of the radio sources is strongly diluted by the broadness of their luminosity functions, implying that objects distributed over distance ranges much larger than the clustering radius contribute to the counts at any flux density. This effect makes their clustering essentially irrelevant in the present context.

Analyses of clustering properties of star-forming galaxies [28, 112] have demonstrated a strong difference between late-types and proto-spheroids. The clustering of the former objects adds a minor contribution to the intensity fluctuations due to point sources, which, in the CORE frequency range, are dominated by the latter population. Ignoring the clustering properties of late-type galaxies is thus a good approximation, since we are only interested in characterizing the fluctuation field; these galaxies have therefore been distributed at random inside the simulated sky area.

In contrast, for proto-spheroidal galaxies we used the method elaborated in Ref. [120], which allowed us to distribute the sources consistently with their clustering properties, as described by the angular power spectrum, $P(k)$. At each frequency we adopted the $P(k \mid \nu)$ given by the model of Ref. [28] that fits the available clustering data on dusty galaxies.

We have used the latest version of the Planck Sky Model [PSM; 121] to check that, in "clean" regions at high Galactic latitudes $\left(|b|>30^{\circ}\right)$, the contribution of Galactic emission to fluctuations at the CORE resolution is small compared to noise and, except at the highest CORE frequencies, to CMB fluctuations. The PSM currently incorporates all the available 
Table 4. Estimated detection limits in polarized flux density and surface densities of sources brighter than such limits for the CORE configuration with a 1-m telescope.

\begin{tabular}{|c|c|c|c|c|c|c|c|c|}
\hline \multirow[b]{2}{*}{$\begin{array}{l}\text { Freq. } \\
{[\mathrm{GHz}]}\end{array}$} & \multirow[b]{2}{*}{$\begin{array}{r}P_{4 \sigma} \\
{[\mathrm{mJy}]}\end{array}$} & \multirow[b]{2}{*}{$\begin{array}{r}P_{5 \sigma} \\
{[\mathrm{mJy}]}\end{array}$} & \multicolumn{2}{|c|}{ Radio } & \multicolumn{2}{|c|}{ Proto-sph } & \multicolumn{2}{|c|}{ Late-type } \\
\hline & & & $\begin{array}{r}N_{4 \sigma} \\
{\left[\mathrm{sr}^{-1}\right]}\end{array}$ & $\begin{array}{r}N_{5 \sigma} \\
{\left[\mathrm{sr}^{-1}\right]}\end{array}$ & $\begin{array}{r}N_{4 \sigma} \\
{\left[\mathrm{sr}^{-1}\right]} \\
\end{array}$ & $\begin{array}{r}N_{5 \sigma} \\
{\left[\mathrm{sr}^{-1}\right]}\end{array}$ & $\begin{array}{r}N_{4 \sigma} \\
{\left[\mathrm{sr}^{-1}\right]} \\
\end{array}$ & $\begin{array}{r}N_{5 \sigma} \\
{\left[\mathrm{sr}^{-1}\right]}\end{array}$ \\
\hline 60 & 11.0 & 13.9 & 91 & 69 & $\ldots$ & $\ldots$ & $\ldots$ & \\
\hline 100 & 9.0 & 13.2 & 99 & 65 & $\ldots$ & $\ldots$ & $\ldots$ & $\ldots$ \\
\hline 145 & 8.0 & 11.7 & 92 & 61 & $\ldots$ & $\ldots$ & $\ldots$ & $\ldots$ \\
\hline 220 & 5.2 & 7.0 & 106 & 77 & 0.3 & 0.1 & 1.7 & 1.1 \\
\hline 340 & 4.5 & 5.8 & 103 & 78 & 17.0 & 6.1 & 20.3 & 13.3 \\
\hline 450 & 5.3 & 7.0 & 79 & 59 & 62.2 & 19.6 & 81.1 & 49.7 \\
\hline 600 & 5.7 & 8.7 & 55 & 34 & 403.4 & 69.7 & 260.5 & 116.9 \\
\hline
\end{tabular}

Table 5. Estimated detection limits in polarized flux density and surface densities of sources brighter than such limits for the CORE configuration with a 1.5-m telescope.

\begin{tabular}{|c|c|c|c|c|c|c|c|c|}
\hline \multirow[b]{2}{*}{$\begin{array}{c}\text { Freq. } \\
\text { [GHz] }\end{array}$} & \multirow[b]{2}{*}{$\begin{array}{r}P_{4 \sigma} \\
\text { [mJy] }\end{array}$} & \multirow[b]{2}{*}{$\begin{array}{r}P_{5 \sigma} \\
{[\mathrm{mJy}]}\end{array}$} & \multicolumn{2}{|c|}{ Radio } & \multicolumn{2}{|c|}{ Proto-sph } & \multicolumn{2}{|c|}{ Late-type } \\
\hline & & & $\begin{array}{r}N_{4 \sigma} \\
{\left[\mathrm{sr}^{-1}\right]}\end{array}$ & $\begin{array}{r}N_{5 \sigma} \\
{\left[\mathrm{sr}^{-1}\right]}\end{array}$ & $\begin{array}{r}N_{4 \sigma} \\
{\left[\mathrm{sr}^{-1}\right]}\end{array}$ & $\begin{array}{r}N_{5 \sigma} \\
{\left[\mathrm{sr}^{-1}\right]}\end{array}$ & $\begin{array}{r}N_{4 \sigma} \\
{\left[\mathrm{sr}^{-1}\right]}\end{array}$ & $\begin{array}{r}N_{5 \sigma} \\
{\left[\mathrm{sr}^{-1}\right]}\end{array}$ \\
\hline 60 & 5.2 & 7.7 & 212 & 137 & $\ldots$ & $\ldots$ & $\ldots$ & $\ldots$ \\
\hline 100 & 5.2 & 6.9 & 184 & 134 & $\ldots$ & $\ldots$ & $\ldots$ & $\ldots$ \\
\hline 145 & 4.6 & 5.6 & 165 & 134 & $\ldots$ & $\ldots$ & $\ldots$ & $\ldots$ \\
\hline 220 & 3.0 & 3.7 & 196 & 154 & 2 & 1 & 4 & 3 \\
\hline 340 & 3.1 & 3.9 & 156 & 122 & 79 & 32 & 40 & 27 \\
\hline 450 & 4.2 & 5.3 & 100 & 78 & 153 & 60 & 123 & 80 \\
\hline 600 & 3.9 & 5.2 & 84 & 61 & 1699 & 596 & 560 & 317 \\
\hline 800 & 4.7 & 6.2 & 78 & 58 & 2215 & 817 & 1581 & 891 \\
\hline
\end{tabular}

information on the various emission components, thus providing a realistic representation of the sky. We have therefore neglected the Galactic emissions. This implies that the detection limits derived below are lower limits for regions with substantial Galactic emission.

As for the CMB, we made use of the PSM software package to generate all-sky intensity and polarization maps in the HEALPix pixelization with high resolution, i.e. with $\mathrm{N}_{\text {side }}=$ 8192 , corresponding to a pixel size of 25.65 arcsec. Regions of the chosen size and angular resolution were projected onto the plane and added to the source maps (that were built on a planar surface). Each map was then filtered with a Gaussian beam function with the appropriated FWHM, and the instrumental noise was added; the adopted values for the FWHM and the noise are specified in Table 3.

We have applied the Mexican-hat wavelet 2 as described in Ref. [122] and [123]. This filtering approach allows an efficient point-source detection and extraction, since it effectively removes CMB structures or extended emission. This cleaning was carried out before attempting any detection. The rms of the final filtered patch was computed after masking all sources detected with signal-to-noise ratio $\mathrm{S} / \mathrm{N}>4$.

In fact the size of patches over which we simulated the source distribution were too small to contain a statistically significant number of sources with polarized flux densities detected with $\mathrm{S} / \mathrm{N}>4$. To determine the detection limits we then injected, at each frequency, fake sources with gradually increasing polarized flux densities. At least five sources per polarized 
flux density bin were introduced in the maps, distributed with a separation at least three times larger than the beam size.

The source detection algorithm was then applied blindly to each of the simulated patches, producing a list of $4 \sigma$ detections. Once the detection limits were determined at each frequency, the surface density of sources brighter than such limits were obtained from the model. The estimated detection limits and number of sources per steradian above them are given in Tables 4 and 5 and illustrated by Fig. 8; results are presented for a set of frequencies in the CORE range and for the 1-m and 1.5-m options.

Objects detected in polarization are essentially only radio sources for frequencies up to around $200 \mathrm{GHz}$. The number of detected dusty galaxies increases with increasing frequency. For the adopted distribution of polarization fraction, CORE will detect similar numbers of radio sources and of dusty galaxies at $\simeq 500 \mathrm{GHz}$. At $600 \mathrm{GHz}$ the latter population will dominate, reaching an integral count of $\simeq 660 \mathrm{sr}^{-1}$ for sources detected with $\mathrm{S} / \mathrm{N}>4$. Of course these estimates are only guesses, since the distribution of the global polarization fraction of dusty galaxies is unknown. We will need the measurements of CORE to probe the polarization distribution.

The CORE150 configuration will do much better, reaching thousands of $\mathrm{S} / \mathrm{N}>4$ detections per steradian in polarization of both proto-spheroidal and late-type galaxies at its highest frequency $(800 \mathrm{GHz})$. As illustrated by Fig. 9 the progress with respect to Planck polarization results is spectacular. In fact, in the "extragalactic zone", Planck detected only a few tens of extragalactic objects in polarisation, all of them radio sources. ${ }^{5}$

Polarization surveys of extragalactic sources are essential to control the contamination of CMB polarimetry experiments with arcmin pixel sizes, and carry crucial information on the source physics. Polarimetric properties of radio-loud AGN at millimetric and sub-millimetric wavelengths tell us about magnetic fields and about the plasma in the inner, unresolved regions of their relativistic jets. Additionally, polarimetry of dusty galaxies as a function of their inclination is informative on the structure and on the ordering of large-scale magnetic fields.

Finally, we note that, like in the total intensity case, CMB-S4 will primarily detect radio sources, reaching deeper flux density levels than CORE. In turn, CORE will extend the frequency coverage and the time-span for variability studies. For studies of polarization properties of dusty galaxies, CORE data will be unique.

\section{Conclusions}

In spite of their sensitivities, now approaching fundamental limits, modern space-borne CMB experiments have provided only shallow surveys of extragalactic sources. The key limitation is source confusion, i.e. intensity peaks due to random positive fluctuations of faint sources that mimic real individual sources. The rms confusion noise scales approximately as the square of the FWHM (see figure 3 of [1]). Hence the substantially better resolution of the diffractionlimited CORE telescope, compared to Planck, especially at the highest frequencies, offers a large advantage in terms of detection limits. The advantage is further boosted in terms of the number of detected sources by the steepness of the source counts.

\footnotetext{
${ }^{5}$ The PCCS2 lists hundreds of detections in polarization, mostly at low Galactic latitudes. The surface densities shown in Fig. 9 are our own estimates based on detections in the "extragalactic zone" above the $90 \%$ completeness limit.
} 
The power of all-sky surveys at $\mathrm{mm}$ and sub-mm wavelengths has already been vividly demonstrated by Planck, which has detected thousands of dusty galaxies as well as many hundreds of extragalactic radio sources in this spectral range, which is difficult or impossible to explore from the ground and only lightly surveyed by other space missions. Planck photometry proved to be crucially important to characterize the synchrotron peak of blazars. Surveys at $\mathrm{mm}$ wavelengths are the most effective way to select this class of sources, which, among other things, constitute the overwhelming majority of the identified extragalactic $\gamma-$ ray sources detected by the Fermi-LAT. Planck data have also provided key information on the energy spectrum of relativistic electrons responsible for the synchrotron emission with interesting implications for their acceleration mechanisms.

Planck detected several of the most extreme, strongly-lensed, high- $z$ galaxies, with estimated gravitational amplifications, $\mu$, of up to 50; CORE will detect thousands of strongly lensed galaxies. Strong lensing offers the opportunity of detailed follow-up studies of high$z$ galaxies with otherwise unattainable sensitivity: the exposure time to reach a given flux density limit varies as $\mu^{-2}$ and, since lensing conserves surface brightness, it stretches the image, thus effectively increasing the angular resolution by a substantial factor (at least in one dimension).

Moreover, Planck proved to be a powerful probe of the evolution of the large-scale structure of the Universe in the phase when early-type galaxies, the dominant population in rich clusters and groups of galaxies, were forming the bulk of their stars.

Beyond strongly expanding the samples of source populations detected by Planck, CORE will open new windows. In particular, it will: (i) provide unbiased, flux limited samples of dense proto-cluster cores of star-forming galaxies, some examples of which were detected by Herschel; (ii) allow a detailed investigation, via direct detections complemented with stacking analysis, of the evolution of the star-formation rate in virialized galaxy clusters detected by surveys of the SZ effect (including those carried out by CORE itself) or by X-ray surveys; (iii) provide the spectrum of the CIB dipole anisotropy, which contains important information on the average CIB intensity spectrum; and (iv) provide the first blind high frequency census of the polarization properties of radio sources and of star-forming galaxies.

\section{Acknowledgments}

Work supported in part by ASI/INAF agreement n. 2014-024-R.1 and by PRIN-INAF 2014 "Probing the AGN/galaxy co-evolution through ultra-deep and ultra-high resolution radio surveys". JGN acknowledges financial support from the Spanish MINECO for a "Ramon y Cajal" fellowship (RYC-2013-13256) and the I+D 2015 project AYA2015-65887-P (MINECO/FEDER). CH-M acknowledges the financial support of the Spanish MINECO through I+D project AYA2015-66211-C2-2-P. MB is supported by the Netherlands Organization for Scientific Research, NWO, through grant number 614.001.451, and by the Polish National Science Centre under contract UMO-2012/07/D/ST9/02785. CJM is supported by an FCT Research Professorship, contract reference IF/00064/2012, funded by FCT/MCTES (Portugal) and POPH/FSE (EC). GR acknowledges support from the National Research Foundation of Korea (NRF) through NRF-SGER 2014055950 funded by the Korean Ministry of Education, Science and Technology (MoEST), and from the faculty research fund of Sejong University in 2016. 


\section{References}

[1] G. De Zotti, G. Castex, J. González-Nuevo, M. Lopez-Caniego, M. Negrello, Z.-Y. Cai, M. Clemens, J. Delabrouille, D. Herranz, L. Bonavera, J.-B. Melin, M. Tucci, S. Serjeant, M. Bilicki, P. Andreani, D. L. Clements, L. Toffolatti, and B. F. Roukema, Extragalactic sources in Cosmic Microwave Background maps, JCAP 6 (June, 2015) 018, [arXiv: 1501.02170$]$.

[2] Planck Collaboration XIII, Planck 2015 results. XIII. Cosmological parameters, A\&3A 594 (Sept., 2016) A13, [arXiv:1502.01589].

[3] J. Delabrouille, P. de Bernardis, M. Bersanelli, F. Bouchet, F. Boulanger, S. Hanani, B. Maffei, D. McCarthy, and et al., "Exploring Cosmic Origins with CORE: Survey requirements and mission design." Feb., 2017.

[4] P. de Bernardis, P. A. R. Ade, J. Baselmans, E. S. Battistelli, A. Benoit, M. Bersanelli, A. Bideaud, M. Calvo, and et al., "Exploring Cosmic Origins with CORE: The Instrument." Feb., 2017.

[5] M. Ashdown, R. Banerji, J. Borrill, A. Buzzelli, G. De Gasperis, J. Delabrouille, E. Hivon, H. D. Thuong, and et al., "Exploring Cosmic Origins with CORE: Mitigation of systematic effects." Feb., 2017.

[6] M. Remazeilles, A. J. Banday, C. Baccigalupi, S. Basak, A. Bonaldi, G. De Zotti, J. Delabrouille, C. Dickinson, and et al., "Exploring Cosmic Origins with CORE: B-mode Component Separation." Feb., 2017.

[7] E. Di Valentino, T. Brinckmann, M. Gerbino, V. Poulin, F. R. Bouchet, J. Lesgourgues, A. Melchiorri, J. Chluba, S. Clesse, J. Delabrouille, C. Dvorkin, F. Forastieri, S. Galli, D. C. Hooper, M. Lattanzi, C. J. A. P. Martins, L. Salvati, G. Cabass, A. Caputo, E. Giusarma, E. Hivon, P. Natoli, L. Pagano, S. Paradiso, J. A. Rubino-Martin, A. Achucarro, M. Ballardini, N. Bartolo, D. Baumann, J. G. Bartlett, P. de Bernardis, A. Bonaldi, M. Bucher, Z.-Y. Cai, G. De Zotti, J. M. Diego, J. Errard, S. Ferraro, F. Finelli, R. T. Genova-Santos, J. Gonzalez-Nuevo, S. Grandis, J. Greenslade, S. Hagstotz, W. Handley, M. Hindmarsh, C. Hernandez-Monteagudo, K. Kiiveri, M. Kunz, A. Lasenby, M. Liguori, M. Lopez-Caniego, G. Luzzi, J.-B. Melin, J. J. Mohr, M. Negrello, D. Paoletti, M. Remazeilles, C. Ringeval, J. Valiviita, B. Van Tent, V. Vennin, N. Vittorio, and the CORE collaboration, Exploring Cosmic Origins with CORE: Cosmological Parameters, ArXiv e-prints (Nov., 2016) [arXiv:1612.00021].

[8] CORE Collaboration, F. Finelli, M. Bucher, A. Achúcarro, M. Ballardini, N. Bartolo, D. Baumann, S. Clesse, J. Errard, W. Handley, M. Hindmarsh, K. Kiiveri, M. Kunz, A. Lasenby, M. Liguori, D. Paoletti, C. Ringeval, J. Väliviita, B. van Tent, V. Vennin, F. Arroja, M. Ashdown, A. J. Banday, R. Banerji, J. Baselmans, J. G. Bartlett, P. de Bernardis, M. Bersanelli, A. Bonaldi, J. Borril, F. R. Bouchet, F. Boulanger, T. Brinckmann, Z.-Y. Cai, M. Calvo, A. Challinor, J. Chluba, G. D’Amico, J. Delabrouille, J. María Diego, G. De Zotti, V. Desjacques, E. Di Valentino, S. Feeney, J. R. Fergusson, S. Ferraro, F. Forastieri, S. Galli, J. García-Bellido, R. T. Génova-Santos, M. Gerbino,

J. González-Nuevo, S. Grandis, J. Greenslade, S. Hagstotz, S. Hanany, D. K. Hazra, C. Hernández-Monteagudo, E. Hivon, B. Hu, E. D. Kovetz, H. Kurki-Suonio, M. Lattanzi, J. Lesgourgues, J. Lizarraga, M. López-Caniego, G. Luzzi, B. Maffei, C. J. A. P. Martins, E. Martínez-González, D. McCarthy, S. Matarrese, A. Melchiorri, J.-B. Melin, A. Monfardini, P. Natoli, M. Negrello, F. Oppizzi, E. Pajer, S. P. Patil, M. Piat, G. Pisano, V. Poulin, A. Ravenni, M. Remazeilles, A. Renzi, D. Roest, L. Salvati, A. Tartari, G. Tasinato, J. Torrado, N. Trappe, M. Tucci, J. Urrestilla, P. Vielva, and R. Van de Weygaert, Exploring Cosmic Origins with CORE: Inflation, ArXiv e-prints (Dec., 2016) [arXiv:1612.08270]. 
[9] J. G. Bartlett, J.-B. Melin, D. Tramonte, J. A. Rubiño-Martin, and et al., "Exploring Cosmic Origins with CORE: Large-Scale Structure Science." Feb., 2017.

[10] J.-B. Melin, A. Bonaldi, M. Remazeilles, S. Hagstotz, J. M. Diego,

C. Hernández-Monteagudo, R.-T. Génova-Santos, G. Luzzi, and et al., "Exploring Cosmic Origins with CORE: Cluster Science." Feb., 2017.

[11] C. Burigana, C. S. Carvalho, T. Trombetti, A. Notari, M. Quartin, G. De Gasperis, A. Buzzelli, N. Vittorio, and et al., "Exploring Cosmic Origins with CORE: implications of observer peculiar motion effects in the CMB." Feb., 2017.

[12] Planck Collaboration XXVI, Planck 2015 results. XXVI. The Second Planck Catalogue of Compact Sources, A\&A 594 (Sept., 2016) A26, [arXiv:1507.02058].

[13] M. Negrello, M. Clemens, J. Gonzalez-Nuevo, G. De Zotti, L. Bonavera, G. Cosco, G. Guarese, L. Boaretto, S. Serjeant, L. Toffolatti, A. Lapi, M. Bethermin, G. Castex, D. L. Clements, J. Delabrouille, H. Dole, A. Franceschini, N. Mandolesi, L. Marchetti, B. Partridge, and A. Sajina, The local luminosity function of star-forming galaxies derived from the Planck Early Release Compact Source Catalogue, MNRAS 429 (Feb., 2013) 1309-1323, [arXiv: 1211.3832].

[14] L. Marchetti, M. Vaccari, A. Franceschini, V. Arumugam, H. Aussel, M. Béthermin, J. Bock, A. Boselli, V. Buat, D. Burgarella, D. L. Clements, A. Conley, L. Conversi, A. Cooray, C. D. Dowell, D. Farrah, A. Feltre, J. Glenn, M. Griffin, E. Hatziminaoglou, S. Heinis, E. Ibar, R. J. Ivison, H. T. Nguyen, B. O'Halloran, S. J. Oliver, M. J. Page, A. Papageorgiou, C. P. Pearson, I. Pérez-Fournon, M. Pohlen, D. Rigopoulou, I. G. Roseboom, M. Rowan-Robinson, B. Schulz, D. Scott, N. Seymour, D. L. Shupe, A. J. Smith, M. Symeonidis, I. Valtchanov, M. Viero, L. Wang, J. Wardlow, C. K. Xu, and M. Zemcov, The HerMES submillimetre local and low-redshift luminosity functions, MNRAS 456 (Feb., 2016) 1999-2023, [arXiv: 1511.06167].

[15] K. Kuehn, J. Lawrence, D. M. Brown, S. Case, M. Colless, R. Content, L. Gers, J. Gilbert, M. Goodwin, A. M. Hopkins, M. Ireland, N. P. F. Lorente, R. Muller, V. Nichani,

A. Rakman, S. N. Richards, W. Saunders, N. F. Staszak, J. Tims, and L. G. Waller, TAIPAN: optical spectroscopy with StarBugs, in Ground-based and Airborne Instrumentation for Astronomy V, vol. 9147 of Proceedings of the SPIE, p. 914710, July, 2014.

[16] J. Comparat, C.-H. Chuang, S. Rodríguez-Torres, M. Pellejero-Ibanez, F. Prada, G. Yepes, H. M. Courtois, G.-B. Zhao, Y. Wang, J. Sanchez, C. Maraston, R. B. Metcalf, J. Peiro-Perez, F. S. Kitaura, E. Pérez, and R. M. González Delgado, The Low Redshift survey at Calar Alto (LoRCA), MNRAS 458 (May, 2016) 2940-2952, [arXiv:1510.00147].

[17] O. Doré, J. Bock, M. Ashby, P. Capak, A. Cooray, R. de Putter, T. Eifler, N. Flagey, Y. Gong, S. Habib, K. Heitmann, C. Hirata, W.-S. Jeong, R. Katti, P. Korngut, E. Krause, D.-H. Lee, D. Masters, P. Mauskopf, G. Melnick, B. Mennesson, H. Nguyen, K. Öberg, A. Pullen, A. Raccanelli, R. Smith, Y.-S. Song, V. Tolls, S. Unwin, T. Venumadhav, M. Viero, M. Werner, and M. Zemcov, Cosmology with the SPHEREX All-Sky Spectral Survey, ArXiv e-prints (Dec., 2014) [arXiv: 1412.4872].

[18] M. Bilicki, T. H. Jarrett, J. A. Peacock, M. E. Cluver, and L. Steward, Two Micron All Sky Survey Photometric Redshift Catalog: A Comprehensive Three-dimensional Census of the Whole Sky, ApJS 210 (Jan., 2014) 9, [arXiv:1311.5246].

[19] M. Bilicki, J. A. Peacock, T. H. Jarrett, M. E. Cluver, N. Maddox, M. J. I. Brown, E. N. Taylor, N. C. Hambly, A. Solarz, B. W. Holwerda, I. Baldry, J. Loveday, A. Moffett, A. M. Hopkins, S. P. Driver, M. Alpaslan, and J. Bland-Hawthorn, WISE $\times$ SuperCOSMOS Photometric Redshift Catalog: 20 Million Galaxies over $3 \pi$ Steradians, ApJS 225 (July, 2016) 5, [arXiv: 1607.01182].

[20] M. López-Caniego, J. González-Nuevo, D. Herranz, M. Massardi, J. L. Sanz, G. De Zotti, L. Toffolatti, and F. Argüeso, Nonblind Catalog of Extragalactic Point Sources from the 
Wilkinson Microwave Anisotropy Probe (WMAP) First 3 Year Survey Data, ApJS 170 (May, 2007) 108-125, [astro-ph/0701473].

[21] K. Małek, A. Pollo, T. T. Takeuchi, V. Buat, D. Burgarella, M. Malkan, E. Giovannoli, A. Kurek, and S. Matsuura, Properties of star forming galaxies in AKARI Deep Field-South, $A \mathscr{E A} 562$ (Feb., 2014) A15, [arXiv:1312.0765].

[22] A. Solarz, A. Pollo, T. T. Takeuchi, K. Małek, H. Matsuhara, G. J. White, A. Pȩpiak, T. Goto, T. Wada, S. Oyabu, T. Takagi, Y. Ohyama, C. P. Pearson, H. Hanami, T. Ishigaki, and M. Malkan, Clustering of the AKARI NEP deep field $24 \mu \mathrm{m}$ selected galaxies, A $6 A \mathbf{5 8 2}$ (Oct., 2015) A58, [arXiv: 1509.00219].

[23] A. Pollo, T. T. Takeuchi, A. Solarz, P. Rybka, T. L. Suzuki, A. Pȩpiak, and S. Oyabu, Clustering of far-infrared galaxies in the AKARI All-Sky Survey North, Earth, Planets, and Space 65 (Oct., 2013) 1109-1116.

[24] K. N. Abazajian, P. Adshead, Z. Ahmed, S. W. Allen, D. Alonso, K. S. Arnold, C. Baccigalupi, J. G. Bartlett, N. Battaglia, B. A. Benson, C. A. Bischoff, J. Borrill, V. Buza, E. Calabrese, R. Caldwell, J. E. Carlstrom, C. L. Chang, T. M. Crawford, F.-Y. Cyr-Racine, F. De Bernardis, T. de Haan, S. di Serego Alighieri, J. Dunkley, C. Dvorkin, J. Errard, G. Fabbian, S. Feeney, S. Ferraro, J. P. Filippini, R. Flauger, G. M. Fuller, V. Gluscevic, D. Green, D. Grin, E. Grohs, J. W. Henning, J. C. Hill, R. Hlozek, G. Holder, W. Holzapfel, W. Hu, K. M. Huffenberger, R. Keskitalo, L. Knox, A. Kosowsky, J. Kovac, E. D. Kovetz, C.-L. Kuo, A. Kusaka, M. Le Jeune, A. T. Lee, M. Lilley, M. Loverde, M. S. Madhavacheril, A. Mantz, D. J. E. Marsh, J. McMahon, P. D. Meerburg, J. Meyers, A. D. Miller, J. B. Munoz, H. N. Nguyen, M. D. Niemack, M. Peloso, J. Peloton, L. Pogosian, C. Pryke, M. Raveri, C. L. Reichardt, G. Rocha, A. Rotti, E. Schaan, M. M. Schmittfull, D. Scott, N. Sehgal, S. Shandera, B. D. Sherwin, T. L. Smith, L. Sorbo, G. D. Starkman, K. T. Story, A. van Engelen, J. D. Vieira, S. Watson, N. Whitehorn, and W. L. Kimmy Wu, CMB-S4 Science Book, First Edition, ArXiv e-prints (Oct., 2016) [arXiv: 1610.02743].

[25] M. Rowan-Robinson and D. L. Clements, Cold galaxies, MNRAS 453 (Oct., 2015) 2050-2057, [arXiv: 1507.08778$]$.

[26] M. Negrello, S. Amber, A. Amvrosiadis, Z.-Y. Cai, A. Lapi, J. Gonzalez-Nuevo, G. De Zotti, C. Furlanetto, S. J. Maddox, M. Allen, T. Bakx, R. S. Bussmann, A. Cooray, G. Covone, L. Danese, H. Dannerbauer, H. Fu, J. Greenslade, M. Gurwell, R. Hopwood, L. V. E. Koopmans, N. Napolitano, H. Nayyeri, A. Omont, C. E. Petrillo, D. A. Riechers, S. Serjeant, C. Tortora, E. Valiante, G. Verdoes Kleijn, G. Vernardos, J. L. Wardlow, M. Baes, A. J. Baker, N. Bourne, D. Clements, S. M. Crawford, S. Dye, L. Dunne, S. Eales, R. J. Ivison, L. Marchetti, M. J. Michałowski, M. W. L. Smith, M. Vaccari, and P. van der Werf, The Herschel-ATLAS: a sample of $500 \mu \mathrm{m}$-selected lensed galaxies over $600 \mathrm{deg}^{2}$, MNRAS 465 (Mar., 2017) 3558-3580, [arXiv:1611.03922].

[27] J. Glenn, A. Conley, M. Béthermin, B. Altieri, A. Amblard, V. Arumugam, H. Aussel, T. Babbedge, A. Blain, J. Bock, A. Boselli, V. Buat, N. Castro-Rodríguez, A. Cava, P. Chanial, D. L. Clements, L. Conversi, A. Cooray, C. D. Dowell, E. Dwek, S. Eales, D. Elbaz, T. P. Ellsworth-Bowers, M. Fox, A. Franceschini, W. Gear, M. Griffin, M. Halpern, E. Hatziminaoglou, E. Ibar, K. Isaak, R. J. Ivison, G. Lagache, G. Laurent, L. Levenson, N. Lu, S. Madden, B. Maffei, G. Mainetti, L. Marchetti, G. Marsden, H. T. Nguyen, B. O’Halloran, S. J. Oliver, A. Omont, M. J. Page, P. Panuzzo, A. Papageorgiou, C. P. Pearson, I. Pérez-Fournon, M. Pohlen, D. Rigopoulou, D. Rizzo, I. G. Roseboom, M. Rowan-Robinson, M. S. Portal, B. Schulz, D. Scott, N. Seymour, D. L. Shupe, A. J. Smith, J. A. Stevens, M. Symeonidis, M. Trichas, K. E. Tugwell, M. Vaccari, I. Valtchanov, J. D. Vieira, L. Vigroux, L. Wang, R. Ward, G. Wright, C. K. Xu, and M. Zemcov, HerMES: deep galaxy number counts from a $P(D)$ fluctuation analysis of SPIRE Science Demonstration Phase observations, MNRAS 409 (Nov., 2010) 109-121, [arXiv:1009.5675]. 
[28] Z.-Y. Cai, A. Lapi, J.-Q. Xia, G. De Zotti, M. Negrello, C. Gruppioni, E. Rigby, G. Castex, J. Delabrouille, and L. Danese, A Hybrid Model for the Evolution of Galaxies and Active Galactic Nuclei in the Infrared, ApJ 768 (May, 2013) 21, [arXiv:1303.2335].

[29] A. Lapi, M. Negrello, J. González-Nuevo, Z.-Y. Cai, G. De Zotti, and L. Danese, Effective Models for Statistical Studies of Galaxy-scale Gravitational Lensing, ApJ 755 (Aug., 2012) 46, [arXiv: 1206.1142].

[30] M. Tucci, L. Toffolatti, G. de Zotti, and E. Martínez-González, High-frequency predictions for number counts and spectral properties of extragalactic radio sources. New evidence of a break at $m m$ wavelengths in spectra of bright blazar sources, A\&A $\mathbf{5 3 3}$ (Sept., 2011) A57, [arXiv:1103.5707].

[31] A. Lapi, J. González-Nuevo, L. Fan, A. Bressan, G. De Zotti, L. Danese, M. Negrello, L. Dunne, S. Eales, S. Maddox, R. Auld, M. Baes, D. G. Bonfield, S. Buttiglione, A. Cava, D. L. Clements, A. Cooray, A. Dariush, S. Dye, J. Fritz, D. Herranz, R. Hopwood, E. Ibar, R. Ivison, M. J. Jarvis, S. Kaviraj, M. López-Caniego, M. Massardi, M. J. Michałowski, E. Pascale, M. Pohlen, E. Rigby, G. Rodighiero, S. Serjeant, D. J. B. Smith, P. Temi, J. Wardlow, and P. van der Werf, Herschel-ATLAS Galaxy Counts and High-redshift Luminosity Functions: The Formation of Massive Early-type Galaxies, ApJ 742 (Nov., 2011) 24, [arXiv:1108.3911].

[32] R. Cañameras, N. P. H. Nesvadba, D. Guery, T. McKenzie, S. König, G. Petitpas, H. Dole, B. Frye, I. Flores-Cacho, L. Montier, M. Negrello, A. Beelen, F. Boone, D. Dicken, G. Lagache, E. Le Floc'h, B. Altieri, M. Béthermin, R. Chary, G. de Zotti, M. Giard, R. Kneissl, M. Krips, S. Malhotra, C. Martinache, A. Omont, E. Pointecouteau, J.-L. Puget, D. Scott, G. Soucail, I. Valtchanov, N. Welikala, and L. Yan, Planck's dusty GEMS: The brightest gravitationally lensed galaxies discovered with the Planck all-sky survey, A\&A $\mathbf{5 8 1}$ (Sept., 2015) A105.

[33] H. Nayyeri, M. Keele, A. Cooray, D. A. Riechers, R. J. Ivison, A. I. Harris, D. T. Frayer, A. J. Baker, S. C. Chapman, S. Eales, D. Farrah, H. Fu, L. Marchetti, R. Marques-Chaves, P. I. Martinez-Navajas, S. J. Oliver, A. Omont, I. Perez-Fournon, D. Scott, M. Vaccari, J. Vieira, M. Viero, L. Wang, and J. Wardlow, Candidate Gravitationally Lensed Dusty Star-forming Galaxies in the Herschel Wide Area Surveys, ApJ 823 (May, 2016) 17, [arXiv:1601.03401].

[34] M. Negrello, F. Perrotta, J. González-Nuevo, L. Silva, G. de Zotti, G. L. Granato, C. Baccigalupi, and L. Danese, Astrophysical and cosmological information from large-scale submillimetre surveys of extragalactic sources, MNRAS 377 (June, 2007) 1557-1568, [astro-ph/0703210].

[35] M. Negrello, R. Hopwood, G. De Zotti, A. Cooray, A. Verma, J. Bock, D. T. Frayer, M. A. Gurwell, A. Omont, R. Neri, H. Dannerbauer, L. L. Leeuw, E. Barton, J. Cooke, S. Kim, E. da Cunha, G. Rodighiero, P. Cox, D. G. Bonfield, M. J. Jarvis, S. Serjeant, R. J. Ivison, S. Dye, I. Aretxaga, D. H. Hughes, E. Ibar, F. Bertoldi, I. Valtchanov, S. Eales, L. Dunne, S. P. Driver, R. Auld, S. Buttiglione, A. Cava, C. A. Grady, D. L. Clements, A. Dariush, J. Fritz, D. Hill, J. B. Hornbeck, L. Kelvin, G. Lagache, M. Lopez-Caniego, J. Gonzalez-Nuevo, S. Maddox, E. Pascale, M. Pohlen, E. E. Rigby, A. Robotham, C. Simpson, D. J. B. Smith, P. Temi, M. A. Thompson, B. E. Woodgate, D. G. York, J. E. Aguirre, A. Beelen, A. Blain, A. J. Baker, M. Birkinshaw, R. Blundell, C. M. Bradford, D. Burgarella, L. Danese, J. S. Dunlop, S. Fleuren, J. Glenn, A. I. Harris, J. Kamenetzky, R. E. Lupu, R. J. Maddalena, B. F. Madore, P. R. Maloney, H. Matsuhara, M. J. Michałowski, E. J. Murphy, B. J. Naylor, H. Nguyen, C. Popescu, S. Rawlings, D. Rigopoulou, D. Scott, K. S. Scott, M. Seibert, I. Smail, R. J. Tuffs, J. D. Vieira, P. P. van der Werf, and J. Zmuidzinas, The Detection of a Population of Submillimeter-Bright, Strongly Lensed Galaxies, Science 330 (Nov., 2010) 800-804, [arXiv:1011.1255].

[36] J. A. Peacock, N. C. Hambly, M. Bilicki, H. T. MacGillivray, L. Miller, M. A. Read, and S. B. 
Tritton, The SuperCOSMOS all-sky galaxy catalogue, MNRAS 462 (Oct., 2016) 2085-2098, [arXiv:1607.01189].

[37] M. F. Skrutskie, R. M. Cutri, R. Stiening, M. D. Weinberg, S. Schneider, J. M. Carpenter, C. Beichman, R. Capps, T. Chester, J. Elias, J. Huchra, J. Liebert, C. Lonsdale, D. G. Monet, S. Price, P. Seitzer, T. Jarrett, J. D. Kirkpatrick, J. E. Gizis, E. Howard, T. Evans, J. Fowler, L. Fullmer, R. Hurt, R. Light, E. L. Kopan, K. A. Marsh, H. L. McCallon, R. Tam, S. Van Dyk, and S. Wheelock, The Two Micron All Sky Survey (2MASS), AJ 131 (Feb., 2006) 1163-1183.

[38] E. L. Wright, P. R. M. Eisenhardt, A. K. Mainzer, M. E. Ressler, R. M. Cutri, T. Jarrett, J. D. Kirkpatrick, D. Padgett, R. S. McMillan, M. Skrutskie, S. A. Stanford, M. Cohen, R. G. Walker, J. C. Mather, D. Leisawitz, T. N. Gautier, III, I. McLean, D. Benford, C. J. Lonsdale, A. Blain, B. Mendez, W. R. Irace, V. Duval, F. Liu, D. Royer, I. Heinrichsen, J. Howard, M. Shannon, M. Kendall, A. L. Walsh, M. Larsen, J. G. Cardon, S. Schick, M. Schwalm, M. Abid, B. Fabinsky, L. Naes, and C.-W. Tsai, The Wide-field Infrared Survey Explorer (WISE): Mission Description and Initial On-orbit Performance, AJ 140 (Dec., 2010) 1868-1881, [arXiv: 1008.0031].

[39] J. J. Condon, W. D. Cotton, E. W. Greisen, Q. F. Yin, R. A. Perley, G. B. Taylor, and J. J. Broderick, The NRAO VLA Sky Survey, AJ 115 (May, 1998) 1693-1716.

[40] T. Mauch, T. Murphy, H. J. Buttery, J. Curran, R. W. Hunstead, B. Piestrzynski, J. G. Robertson, and E. M. Sadler, SUMSS: a wide-field radio imaging survey of the southern skyII. The source catalogue, MNRAS 342 (July, 2003) 1117-1130, [astro-ph/0303188].

[41] S. A. Eales, Practical cosmology with lenses, MNRAS 446 (Jan., 2015) 3224-3234.

[42] X.-L. Meng, T. Treu, A. Agnello, M. W. Auger, K. Liao, and P. J. Marshall, Precision cosmology with time delay lenses: high resolution imaging requirements, JCAP 9 (Sept., 2015) 059, [arXiv: 1506.07640].

[43] S. Serjeant, Strong Gravitational Lenses and Multi-Wavelength Galaxy Surveys with AKARI, Herschel, SPICA and Euclid, ArXiv e-prints (Apr., 2016) [arXiv:1604.00282].

[44] P. P. van der Werf, A. Berciano Alba, M. Spaans, A. F. Loenen, R. Meijerink, D. A. Riechers, P. Cox, A. Weiß, and F. Walter, Water Vapor Emission Reveals a Highly Obscured, Star-forming Nuclear Region in the QSO Host Galaxy APM 08279+5255 at z=3.9, ApJL 741 (Nov., 2011) L38, [arXiv:1106.4825].

[45] R. J. Ivison, A. M. Swinbank, I. Smail, A. I. Harris, R. S. Bussmann, A. Cooray, P. Cox, H. Fu, A. Kovács, M. Krips, D. Narayanan, M. Negrello, R. Neri, J. Peñarrubia, J. Richard, D. A. Riechers, K. Rowlands, J. G. Staguhn, T. A. Targett, S. Amber, A. J. Baker, N. Bourne, F. Bertoldi, M. Bremer, J. A. Calanog, D. L. Clements, H. Dannerbauer, A. Dariush, G. De Zotti, L. Dunne, S. A. Eales, D. Farrah, S. Fleuren, A. Franceschini, J. E. Geach, R. D. George, J. C. Helly, R. Hopwood, E. Ibar, M. J. Jarvis, J.-P. Kneib, S. Maddox, A. Omont, D. Scott, S. Serjeant, M. W. L. Smith, M. A. Thompson, E. Valiante, I. Valtchanov, J. Vieira, and P. van der Werf, Herschel-ATLAS: A Binary HyLIRG Pinpointing a Cluster of Starbursting Protoellipticals, ApJ 772 (Aug., 2013) 137, [arXiv: 1302.4436].

[46] T. Wang, D. Elbaz, E. Daddi, A. Finoguenov, D. Liu, C. Schreiber, S. Martín, V. Strazzullo, F. Valentino, R. van der Burg, A. Zanella, L. Ciesla, R. Gobat, A. Le Brun, M. Pannella, M. Sargent, X. Shu, Q. Tan, N. Cappelluti, and Y. Li, Discovery of a Galaxy Cluster with a Violently Starbursting Core at $z=2.506$, ApJ 828 (Sept., 2016) 56, [arXiv:1604.07404].

[47] D. L. Clements, F. G. Braglia, A. K. Hyde, I. Pérez-Fournon, J. Bock, A. Cava, S. Chapman, A. Conley, A. Cooray, D. Farrah, E. A. González Solares, L. Marchetti, G. Marsden, S. J. Oliver, I. G. Roseboom, B. Schulz, A. J. Smith, M. Vaccari, J. Vieira, M. Viero, L. Wang, J. Wardlow, M. Zemcov, and G. de Zotti, Herschel Multitiered Extragalactic Survey: clusters 
of dusty galaxies uncovered by Herschel and Planck, MNRAS 439 (Apr., 2014) 1193-1211, [arXiv: 1311.5758].

[48] M. Negrello, J. González-Nuevo, M. Magliocchetti, L. Moscardini, G. De Zotti, L. Toffolatti, and L. Danese, Effect of clustering on extragalactic source counts with low-resolution instruments, MNRAS 358 (Apr., 2005) 869-874, [astro-ph/0406388].

[49] Planck Collaboration XXXIX, Planck intermediate results. XXXIX. The Planck list of high-redshift source candidates, AESA 596 (Dec., 2016) A100, [arXiv:1508.04171].

[50] Planck Collaboration XXVII, Planck intermediate results. XXVII. High-redshift infrared galaxy overdensity candidates and lensed sources discovered by Planck and confirmed by Herschel-SPIRE, A 6 A 582 (Oct., 2015) A30, [arXiv:1503.08773].

[51] R. C. Kennicutt and N. J. Evans, Star Formation in the Milky Way and Nearby Galaxies, ARAEA 50 (Sept., 2012) 531-608, [arXiv:1204.3552].

[52] R. Aversa, A. Lapi, G. de Zotti, F. Shankar, and L. Danese, Black Hole and Galaxy Coevolution from Continuity Equation and Abundance Matching, ApJ 810 (Sept., 2015) 74, [arXiv: 1507.07318].

[53] I. Flores-Cacho, D. Pierini, G. Soucail, L. Montier, H. Dole, E. Pointecouteau, R. Pelló, E. Le Floc'h, N. Nesvadba, G. Lagache, D. Guery, and R. Cañameras, Multi-wavelength characterisation of $z \sim 2$ clustered, dusty star-forming galaxies discovered by Planck, A $\mho A \mathbf{5 8 5}$ (Jan., 2016) A54, [arXiv:1510.01585].

[54] S. Eales, L. Dunne, D. Clements, A. Cooray, G. De Zotti, S. Dye, R. Ivison, M. Jarvis, G. Lagache, S. Maddox, M. Negrello, S. Serjeant, M. A. Thompson, E. Van Kampen, A. Amblard, P. Andreani, M. Baes, A. Beelen, G. J. Bendo, D. Benford, F. Bertoldi, J. Bock, D. Bonfield, A. Boselli, C. Bridge, V. Buat, D. Burgarella, R. Carlberg, A. Cava, P. Chanial, S. Charlot, N. Christopher, P. Coles, L. Cortese, A. Dariush, E. da Cunha, G. Dalton, L. Danese, H. Dannerbauer, S. Driver, J. Dunlop, L. Fan, D. Farrah, D. Frayer, C. Frenk, J. Geach, J. Gardner, H. Gomez, J. González-Nuevo, E. González-Solares, M. Griffin, M. Hardcastle, E. Hatziminaoglou, D. Herranz, D. Hughes, E. Ibar, W.-S. Jeong, C. Lacey, A. Lapi, A. Lawrence, M. Lee, L. Leeuw, J. Liske, M. López-Caniego, T. Müller, K. Nandra, P. Panuzzo, A. Papageorgiou, G. Patanchon, J. Peacock, C. Pearson, S. Phillipps, M. Pohlen, C. Popescu, S. Rawlings, E. Rigby, M. Rigopoulou, A. Robotham, G. Rodighiero, A. Sansom, B. Schulz, D. Scott, D. J. B. Smith, B. Sibthorpe, I. Smail, J. Stevens, W. Sutherland, T. Takeuchi, J. Tedds, P. Temi, R. Tuffs, M. Trichas, M. Vaccari, I. Valtchanov, P. van der Werf, A. Verma, J. Vieria, C. Vlahakis, and G. J. White, The Herschel ATLAS, PASP 122 (May, 2010) 499-515, [arXiv: 0910.4279].

[55] S. J. Oliver, J. Bock, B. Altieri, A. Amblard, V. Arumugam, H. Aussel, T. Babbedge, A. Beelen, M. Béthermin, A. Blain, A. Boselli, C. Bridge, D. Brisbin, V. Buat, D. Burgarella, N. Castro-Rodríguez, A. Cava, P. Chanial, M. Cirasuolo, D. L. Clements, A. Conley, L. Conversi, A. Cooray, C. D. Dowell, E. N. Dubois, E. Dwek, S. Dye, S. Eales, D. Elbaz, D. Farrah, A. Feltre, P. Ferrero, N. Fiolet, M. Fox, A. Franceschini, W. Gear, E. Giovannoli, J. Glenn, Y. Gong, E. A. González Solares, M. Griffin, M. Halpern, M. Harwit, E. Hatziminaoglou, S. Heinis, P. Hurley, H. S. Hwang, A. Hyde, E. Ibar, O. Ilbert, K. Isaak, R. J. Ivison, G. Lagache, E. Le Floc'h, L. Levenson, B. L. Faro, N. Lu, S. Madden, B. Maffei, G. Magdis, G. Mainetti, L. Marchetti, G. Marsden, J. Marshall, A. M. J. Mortier, H. T. Nguyen, B. O'Halloran, A. Omont, M. J. Page, P. Panuzzo, A. Papageorgiou, H. Patel, C. P. Pearson, I. Pérez-Fournon, M. Pohlen, J. I. Rawlings, G. Raymond, D. Rigopoulou, L. Riguccini, D. Rizzo, G. Rodighiero, I. G. Roseboom, M. Rowan-Robinson, M. Sánchez Portal, B. Schulz, D. Scott, N. Seymour, D. L. Shupe, A. J. Smith, J. A. Stevens, M. Symeonidis, M. Trichas, K. E. Tugwell, M. Vaccari, I. Valtchanov, J. D. Vieira, M. Viero, L. Vigroux, L. Wang, R. Ward, J. Wardlow, G. Wright, C. K. Xu, and M. Zemcov, The 
Herschel Multi-tiered Extragalactic Survey: HerMES, MNRAS 424 (Aug., 2012) 1614-1635, [arXiv: 1203.2562].

[56] G. L. Granato, C. Ragone-Figueroa, R. Domínguez-Tenreiro, A. Obreja, S. Borgani, G. De Lucia, and G. Murante, The early phases of galaxy clusters formation in IR: coupling hydrodynamical simulations with GRASIL-3D, MNRAS 450 (June, 2015) 1320-1332, [arXiv: 1412.6105].

[57] S. Alberts, A. Pope, M. Brodwin, D. W. Atlee, Y.-T. Lin, A. Dey, P. R. M. Eisenhardt, D. P. Gettings, A. H. Gonzalez, B. T. Jannuzi, C. L. Mancone, J. Moustakas, G. F. Snyder, S. A. Stanford, D. Stern, B. J. Weiner, and G. R. Zeimann, The evolution of dust-obscured star formation activity in galaxy clusters relative to the field over the last 9 billion years, MNRAS 437 (Jan., 2014) 437-457, [arXiv: 1310.6040].

[58] S. Alberts, A. Pope, M. Brodwin, S. M. Chung, R. Cybulski, A. Dey, P. R. M. Eisenhardt, A. Galametz, A. H. Gonzalez, B. T. Jannuzi, S. A. Stanford, G. F. Snyder, D. Stern, and G. R. Zeimann, Star Formation and AGN Activity in Galaxy Clusters from $z=1-2:$ a Multi-Wavelength Analysis Featuring Herschel/PACS, ApJ 825 (July, 2016) 72, [arXiv: 1604.03564].

[59] C. R. Wagner, S. Courteau, M. Brodwin, S. A. Stanford, G. F. Snyder, and D. Stern, The Evolution of Star formation Activity in Cluster Galaxies over $0.15<z<1.5$, ApJ 834 (Jan., 2017) 53, [arXiv: 1610.01498].

[60] N. Mehrtens, A. K. Romer, M. Hilton, E. J. Lloyd-Davies, C. J. Miller, S. A. Stanford, M. Hosmer, B. Hoyle, C. A. Collins, A. R. Liddle, P. T. P. Viana, R. C. Nichol, J. P. Stott, E. N. Dubois, S. T. Kay, M. Sahlén, O. Young, C. J. Short, L. Christodoulou, W. A. Watson, M. Davidson, C. D. Harrison, L. Baruah, M. Smith, C. Burke, J. A. Mayers, P.-J. Deadman, P. J. Rooney, E. M. Edmondson, M. West, H. C. Campbell, A. C. Edge, R. G. Mann, K. Sabirli, D. Wake, C. Benoist, L. da Costa, M. A. G. Maia, and R. Ogando, The XMM Cluster Survey: optical analysis methodology and the first data release, MNRAS 423 (June, 2012) 1024-1052, [arXiv: 1106.3056].

[61] A. Merloni, P. Predehl, W. Becker, H. Böhringer, T. Boller, H. Brunner, M. Brusa, K. Dennerl, M. Freyberg, P. Friedrich, A. Georgakakis, F. Haberl, G. Hasinger, N. Meidinger, J. Mohr, K. Nandra, A. Rau, T. H. Reiprich, J. Robrade, M. Salvato, A. Santangelo, M. Sasaki, A. Schwope, J. Wilms, and t. German eROSITA Consortium, eROSITA Science Book: Mapping the Structure of the Energetic Universe, ArXiv e-prints (Sept., 2012) [arXiv:1209.3114].

[62] H. Böhringer, G. Chon, C. A. Collins, L. Guzzo, N. Nowak, and S. Bobrovskyi, The extended ROSAT-ESO flux limited X-ray galaxy cluster survey (REFLEX II) II. Construction and properties of the survey, A\&A $\mathbf{5 5 5}$ (July, 2013) A30.

[63] M. Hasselfield, M. Hilton, T. A. Marriage, G. E. Addison, L. F. Barrientos, N. Battaglia, E. S. Battistelli, J. R. Bond, D. Crichton, S. Das, M. J. Devlin, S. R. Dicker, J. Dunkley, R. Dünner, J. W. Fowler, M. B. Gralla, A. Hajian, M. Halpern, A. D. Hincks, R. Hlozek, J. P. Hughes, L. Infante, K. D. Irwin, A. Kosowsky, D. Marsden, F. Menanteau, K. Moodley, M. D. Niemack, M. R. Nolta, L. A. Page, B. Partridge, E. D. Reese, B. L. Schmitt, N. Sehgal, B. D. Sherwin, J. Sievers, C. Sifón, D. N. Spergel, S. T. Staggs, D. S. Swetz, E. R. Switzer, R. Thornton, H. Trac, and E. J. Wollack, The Atacama Cosmology Telescope: Sunyaev-Zel'dovich selected galaxy clusters at $148 \mathrm{GHz}$ from three seasons of data, JCAP $\mathbf{7}$ (July, 2013) 008, [arXiv: 1301.0816].

[64] L. E. Bleem, B. Stalder, T. de Haan, K. A. Aird, S. W. Allen, D. E. Applegate, M. L. N. Ashby, M. Bautz, M. Bayliss, B. A. Benson, S. Bocquet, M. Brodwin, J. E. Carlstrom, C. L. Chang, and et al., Galaxy Clusters Discovered via the Sunyaev-Zel'dovich Effect in the 2500-Square-Degree SPT-SZ Survey, ApJS 216 (Feb., 2015) 27, [arXiv:1409.0850]. 
[65] Planck Collaboration XXXII, Planck 2013 results. XXXII. The updated Planck catalogue of Sunyaev-Zeldovich sources, A\&A 581 (Sept., 2015) A14, [arXiv:1502.00543].

[66] Planck Collaboration XLIII, Planck intermediate results. XLIII. Spectral energy distribution of dust in clusters of galaxies, A\&A 596 (Dec., 2016) A104, [arXiv: 1603.04919].

[67] Planck Collaboration XXIII, Planck 2015 results. XXIII. The thermal Sunyaev-Zeldovich effect-cosmic infrared background correlation, A\&3A 594 (Aug., 2016) A23, [arXiv: 1509.06555].

[68] P. Popesso, A. Biviano, A. Finoguenov, D. Wilman, M. Salvato, B. Magnelli, C. Gruppioni, F. Pozzi, G. Rodighiero, F. Ziparo, S. Berta, D. Elbaz, M. Dickinson, D. Lutz, B. Altieri, H. Aussel, A. Cimatti, D. Fadda, O. Ilbert, E. Le Floch, R. Nordon, A. Poglitsch, and C. K. $\mathrm{Xu}$, The evolution of galaxy star formation activity in massive haloes, AछA $\mathbf{5 7 4}$ (Feb., 2015) A105, [arXiv: 1407.8214].

[69] C. Gruppioni, F. Pozzi, G. Rodighiero, I. Delvecchio, S. Berta, L. Pozzetti, G. Zamorani, P. Andreani, A. Cimatti, O. Ilbert, E. Le Floc'h, D. Lutz, and et al., The Herschel PEP/HerMES luminosity function - I. Probing the evolution of PACS selected Galaxies to $z \simeq 4$, MNRAS 432 (June, 2013) 23-52, [arXiv:1302.5209].

[70] M. Brodwin, S. A. Stanford, A. H. Gonzalez, G. R. Zeimann, G. F. Snyder, C. L. Mancone, A. Pope, P. R. Eisenhardt, D. Stern, S. Alberts, M. L. N. Ashby, M. J. I. Brown, R.-R. Chary, A. Dey, A. Galametz, D. P. Gettings, B. T. Jannuzi, E. D. Miller, J. Moustakas, and L. A. Moustakas, The Era of Star Formation in Galaxy Clusters, ApJ 779 (Dec., 2013) 138, [arXiv:1310.6039].

[71] T. A. Enßlin and C. R. Kaiser, Comptonization of the cosmic microwave background by relativistic plasma, A\&A 360 (Aug., 2000) 417-430, [astro-ph/0001429].

[72] S. Colafrancesco, P. Marchegiani, and R. Buonanno, Untangling the atmosphere of the Bullet cluster with Sunyaev-Zeldovich effect observations, A $\circlearrowleft$ A 527 (Mar., 2011) L1.

[73] Planck Collaboration XVIII, Planck early results. XVIII. The power spectrum of cosmic infrared background anisotropies, A\&A 536 (Dec., 2011) A18, [arXiv:1101.2028].

[74] Planck Collaboration XXX, Planck 2013 results. XXX. Cosmic infrared background measurements and implications for star formation, A\&A $\mathbf{5 7 1}$ (Nov., 2014) A30, [arXiv: 1309.0382].

[75] D. S. Y. Mak, A. Challinor, G. Efstathiou, and G. Lagache, Measurement of CIB power spectra over large sky areas from Planck HFI maps, MNRAS 466 (Apr., 2017) 286-319, [arXiv: 1609.08942].

[76] Planck Collaboration XLVIII, Planck intermediate results. XLVIII. Disentangling Galactic dust emission and cosmic infrared background anisotropies, A\&A 596 (Dec., 2016) A109, [arXiv: 1605.09387].

[77] H.-Y. Wu and O. Doré, Optimizing future experiments of cosmic far-infrared background: a principal component approach, ArXiv e-prints (Dec., 2016) [arXiv:1612.02474].

[78] M. Tucci, V. Desjacques, and M. Kunz, Cosmic infrared background anisotropies as a window into primordial non-Gaussianity, MNRAS 463 (Dec., 2016) 2046-2063, [arXiv: 1606. 02323].

[79] L. Danese and G. de Zotti, Dipole anisotropy and distortions of the spectrum of the cosmic microwave background, A\&\&A 94 (Feb., 1981) L33.

[80] M. Piat, G. Lagache, J. P. Bernard, M. Giard, and J. L. Puget, Cosmic background dipole measurements with the Planck-High Frequency Instrument, A\&A 393 (Oct., 2002) 359-368, [astro-ph/0110650].

[81] S. A. Balashev, E. E. Kholupenko, J. Chluba, A. V. Ivanchik, and D. A. Varshalovich, Spectral Distortions of the CMB Dipole, ApJ 810 (Sept., 2015) 131, [arXiv:1505.06028]. 
[82] G. De Zotti, M. Negrello, G. Castex, A. Lapi, and M. Bonato, Another look at distortions of the Cosmic Microwave Background spectrum, JCAP 3 (Mar., 2016) 047, [arXiv:1512.04816].

[83] D. J. Fixsen, E. Dwek, J. C. Mather, C. L. Bennett, and R. A. Shafer, The Spectrum of the Extragalactic Far-Infrared Background from the COBE FIRAS Observations, ApJ 508 (Nov., 1998) 123-128, [astro-ph/9803021].

[84] D. J. Fixsen and A. Kashlinsky, Probing the Universe's Tilt with the Cosmic Infrared Background Dipole, ApJ 734 (June, 2011) 61, [arXiv: 1104.0901].

[85] K. Basu, C. Hernández-Monteagudo, and R. A. Sunyaev, CMB observations and the production of chemical elements at the end of the dark ages, A\&A 416 (Mar., 2004) 447-466, [astro-ph/0311620].

[86] C. Hernández-Monteagudo, J. A. Rubiño-Martín, and R. A. Sunyaev, On the influence of resonant scattering on cosmic microwave background polarization anisotropies, MNRAS $\mathbf{3 8 0}$ (Oct., 2007) 1656-1668.

[87] C. Hernández-Monteagudo, L. Verde, and R. Jimenez, Tomography of the Reionization Epoch with Multifrequency CMB Observations, ApJ 653 (Dec., 2006) 1-10, [astro-ph/0604324].

[88] A. Kogut, J. Chluba, D. J. Fixsen, S. Meyer, and D. Spergel, The Primordial Inflation Explorer (PIXIE), in Society of Photo-Optical Instrumentation Engineers (SPIE) Conference Series, vol. 9904 of Proceedings of the SPIE, p. 99040W, July, 2016.

[89] M. Righi, C. Hernández-Monteagudo, and R. A. Sunyaev, Carbon monoxide line emission as a CMB foreground: tomography of the star-forming universe with different spectral resolutions, A\&A 489 (Oct., 2008) 489-504, [arXiv:0805.2174].

[90] N. Mashian, A. Loeb, and A. Sternberg, Spectral distortion of the CMB by the cumulative CO emission from galaxies throughout cosmic history, MNRAS 458 (May, 2016) L99-L103, [arXiv: 1601.02618].

[91] C. Hernández-Monteagudo, Z. Haiman, R. Jimenez, and L. Verde, Oxygen Pumping: Probing Intergalactic Metals at the Epoch of Reionization, ApJL 660 (May, 2007) L85-L88.

[92] C. Hernández-Monteagudo, Z. Haiman, L. Verde, and R. Jimenez, Oxygen Pumping. II. Probing the Inhomogeneous Metal Enrichment at the Epoch of Reionization with High-Frequency CMB Observations, ApJ 672 (Jan., 2008) 33-39, [arXiv:0709.3313].

[93] T. R. Greve, I. Leonidaki, E. M. Xilouris, A. Weiß, Z.-Y. Zhang, P. van der Werf, S. Aalto, L. Armus, T. Díaz-Santos, A. S. Evans, J. Fischer, Y. Gao, E. González-Alfonso, A. Harris, C. Henkel, R. Meijerink, D. A. Naylor, H. A. Smith, M. Spaans, G. J. Stacey, S. Veilleux, and F. Walter, Star Formation Relations and CO Spectral Line Energy Distributions across the J-ladder and Redshift, ApJ $\mathbf{7 9 4}$ (Oct., 2014) 142, [arXiv: 1407.4400].

[94] M. Bonato, M. Negrello, Z.-Y. Cai, G. De Zotti, A. Bressan, A. Lapi, C. Gruppioni, L. Spinoglio, and L. Danese, Exploring the early dust-obscured phase of galaxy formation with blind mid-/far-infrared spectroscopic surveys, MNRAS 438 (Mar., 2014) 2547-2564, [arXiv: 1312.1891].

[95] C. M. Urry and P. Padovani, Unified Schemes for Radio-Loud Active Galactic Nuclei, PASP 107 (Sept., 1995) 803, [astro-ph/9506063].

[96] F. Acero, M. Ackermann, M. Ajello, A. Albert, W. B. Atwood, M. Axelsson, L. Baldini, J. Ballet, , and Fermi-LAT Collaboration, Fermi Large Area Telescope Third Source Catalog, ApJS 218 (June, 2015) 23, [arXiv: 1501.02003].

[97] G. de Zotti, R. Ricci, D. Mesa, L. Silva, P. Mazzotta, L. Toffolatti, and J. González-Nuevo, Predictions for high-frequency radio surveys of extragalactic sources, A\&A 431 (Mar., 2005) 893-903, [astro-ph/0410709]. 
[98] M. Massardi, A. Bonaldi, M. Negrello, S. Ricciardi, A. Raccanelli, and G. de Zotti, A model for the cosmological evolution of low-frequency radio sources, MNRAS 404 (May, 2010) 532-544, [arXiv: 1001.1069].

[99] L. L. Cowie, A. Songaila, E. M. Hu, and J. G. Cohen, New Insight on Galaxy Formation and Evolution From Keck Spectroscopy of the Hawaii Deep Fields, AJ 112 (Sept., 1996) 839, [astro-ph/9606079].

[100] E. E. Rigby, J. Argyle, P. N. Best, D. Rosario, and H. J. A. Röttgering, Cosmic downsizing of powerful radio galaxies to low radio luminosities, A\&A $\mathbf{5 8 1}$ (Sept., 2015) A96, [arXiv: 1507.00341].

[101] Planck Collaboration XV, Planck early results. XV. Spectral energy distributions and radio continuum spectra of northern extragalactic radio sources, A\&B 536 (Dec., 2011) A15, [arXiv: 1101.2047].

[102] Planck Collaboration XLV, Planck intermediate results. XLV. Radio spectra of northern extragalactic radio sources, A\&A 596 (Dec., 2016) A106, [arXiv:1606.05120].

[103] M. Massardi, A. Bonaldi, L. Bonavera, G. De Zotti, M. Lopez-Caniego, and V. Galluzzi, The Planck-ATCA Co-eval Observations project: analysis of radio source properties between 5 and 217 GHz, MNRAS 455 (Jan., 2016) 3249-3262, [arXiv:1511.02605].

[104] S. Cutini, S. Ciprini, M. Orienti, A. Tramacere, F. D'Ammando, F. Verrecchia, G. Polenta, L. Carrasco, V. D’Elia, P. Giommi, J. González-Nuevo, P. Grandi, D. Harrison, E. Hays, S. Larsson, A. Lähteenmäki, J. León-Tavares, M. López-Caniego, P. Natoli, R. Ojha, B. Partridge, A. Porras, L. Reyes, E. Recillas, and E. Torresi, Radio-gamma-ray connection and spectral evolution in $4 C+49.22(S 41150+49)$ : the Fermi, Swift and Planck view, MNRAS 445 (Dec., 2014) 4316-4334, [arXiv:1409.8101].

[105] G. de Zotti, M. Massardi, M. Negrello, and J. Wall, Radio and millimeter continuum surveys and their astrophysical implications, A\&A Rev. 18 (Feb., 2010) 1-65, [arXiv:0908.1896].

[106] L. M. Mocanu, T. M. Crawford, J. D. Vieira, K. A. Aird, M. Aravena, J. E. Austermann, B. A. Benson, M. Béthermin, L. E. Bleem, and et al., Extragalactic Millimeter-wave Point-source Catalog, Number Counts and Statistics from $771 \mathrm{deg}^{2}$ of the SPT-SZ Survey, ApJ 779 (Dec., 2013) 61, [arXiv: 1306. 3470].

[107] D. Marsden, M. Gralla, T. A. Marriage, E. R. Switzer, B. Partridge, M. Massardi, G. Morales, G. Addison, J. R. Bond, D. Crichton, S. Das, M. Devlin, R. Dünner, A. Hajian, M. Hilton, A. Hincks, J. P. Hughes, K. Irwin, A. Kosowsky, F. Menanteau, K. Moodley, M. Niemack, L. Page, E. D. Reese, B. Schmitt, N. Sehgal, J. Sievers, S. Staggs, D. Swetz, R. Thornton, and E. Wollack, The Atacama Cosmology Telescope: dusty star-forming galaxies and active galactic nuclei in the Southern survey, MNRAS 439 (Apr., 2014) 1556-1574, [arXiv: 1306.2288].

[108] Planck Collaboration XIII, Planck early results. XIII. Statistical properties of extragalactic radio sources in the Planck Early Release Compact Source Catalogue, A\&SA 536 (Dec., 2011) A13, [arXiv: 1101.2044].

[109] P. Giommi, G. Polenta, A. Lähteenmäki, D. J. Thompson, M. Capalbi, S. Cutini, D. Gasparrini, J. González-Nuevo, J. León-Tavares, M. López-Caniego, M. N. Mazziotta, and et al., Simultaneous Planck, Swift, and Fermi observations of X-ray and $\gamma$-ray selected blazars, $A \& A 541$ (May, 2012) A160, [arXiv:1108.1114].

[110] J. León-Tavares, E. Valtaoja, P. Giommi, G. Polenta, M. Tornikoski, A. Lähteenmäki, D. Gasparrini, and S. Cutini, Exploring the Relation between (Sub-)Millimeter Radiation and

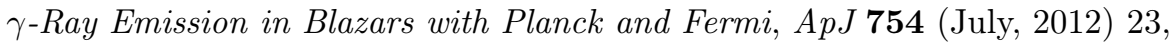
[arXiv: 1204.3589]. 
[111] J. P. Rachen, L. Fuhrmann, T. Krichbaum, E. Angelakis, I. Nestoras, A. Zensus, A. Sievers, H. Ungerechts, E. Keihänen, and M. Reinecke, Coeval Observations of a Complete Sample of Blazars with Effelsberg, IRAM 30m, and Planck, ArXiv e-prints (Mar., 2016) [arXiv: 1603.02144].

[112] J.-Q. Xia, M. Negrello, A. Lapi, G. De Zotti, L. Danese, and M. Viel, Clustering of submillimetre galaxies in a self-regulated baryon collapse model, MNRAS 422 (May, 2012) 1324-1331, [arXiv: 1111.4212].

[113] M. Massardi, S. G. Burke-Spolaor, T. Murphy, R. Ricci, M. López-Caniego, M. Negrello, R. Chhetri, G. De Zotti, R. D. Ekers, R. B. Partridge, and E. M. Sadler, A polarization survey of bright extragalactic AT20G sources, MNRAS 436 (Dec., 2013) 2915-2928, [arXiv: 1309.2527].

[114] M. Tucci and L. Toffolatti, The Impact of Polarized Extragalactic Radio Sources on the Detection of CMB Anisotropies in Polarization, Advances in Astronomy 2012 (Dec., 2012) 624987, [arXiv: 1204.0427].

[115] V. Galluzzi and M. Massardi, The polarimetric multi-frequency radio sources properties, International Journal of Modern Physics D 25 (Mar., 2016) 1640005, [arXiv:1611.08159].

[116] R. A. Battye, I. W. A. Browne, M. W. Peel, N. J. Jackson, and C. Dickinson, Statistical properties of polarized radio sources at high frequency and their impact on cosmic microwave background polarization measurements, MNRAS 413 (May, 2011) 132-148, [arXiv: 1003.5846].

[117] L. Bonavera, J. González-Nuevo, F. Argüeso, and L. Toffolatti, Statistics of the fractional polarisation of compact radio sources in Planck maps, ArXiv e-prints (Mar., 2017) [arXiv: 1703.09952].

[118] J. S. Greaves and W. S. Holland, Submillimetre polarization of M82 and the Galactic Center: Implications for CMB polarimetry, in Astrophysical Polarized Backgrounds (S. Cecchini, S. Cortiglioni, R. Sault, and C. Sbarra, eds.), vol. 609 of American Institute of Physics Conference Series, pp. 267-270, Mar., 2002.

[119] B. C. Matthews, C. A. McPhee, L. M. Fissel, and R. L. Curran, The Legacy of SCUPOL: 850 um Imaging Polarimetry from 1997 to 2005, ApJS 182 (May, 2009) 143-204.

[120] J. González-Nuevo, L. Toffolatti, and F. Argüeso, Predictions of the Angular Power Spectrum of Clustered Extragalactic Point Sources at Cosmic Microwave Background Frequencies from Flat and All-Sky Two-dimensional Simulations, ApJ 621 (Mar., 2005) 1-14, [astro-ph/0405553].

[121] J. Delabrouille, M. Betoule, J.-B. Melin, M.-A. Miville-Deschênes, J. Gonzalez-Nuevo, M. Le Jeune, G. Castex, G. de Zotti, S. Basak, M. Ashdown, J. Aumont, C. Baccigalupi, A. J. Banday, J.-P. Bernard, F. R. Bouchet, D. L. Clements, A. da Silva, C. Dickinson, F. Dodu, K. Dolag, F. Elsner, L. Fauvet, G. Fä̈, G. Giardino, S. Leach, J. Lesgourgues, M. Liguori, J. F. Macías-Pérez, M. Massardi, S. Matarrese, P. Mazzotta, L. Montier, S. Mottet, R. Paladini, B. Partridge, R. Piffaretti, G. Prezeau, S. Prunet, S. Ricciardi, M. Roman, B. Schaefer, and L. Toffolatti, The pre-launch Planck Sky Model: a model of sky emission at submillimetre to centimetre wavelengths, A\&A 553 (May, 2013) A96, [arXiv:1207.3675].

[122] J. González-Nuevo, F. Argüeso, M. López-Caniego, L. Toffolatti, J. L. Sanz, P. Vielva, and D. Herranz, The Mexican hat wavelet family: application to point-source detection in cosmic microwave background maps, MNRAS 369 (July, 2006) 1603-1610, [astro-ph/0604376].

[123] M. López-Caniego, D. Herranz, J. González-Nuevo, J. L. Sanz, R. B. Barreiro, P. Vielva, F. Argüeso, and L. Toffolatti, Comparison of filters for the detection of point sources in Planck simulations, MNRAS 370 (Aug., 2006) 2047-2063, [astro-ph/0606199]. 